\title{
Collider tests of the Renormalizable Coloron Model
}

\author{
Yang Bai ${ }^{a}$ and Bogdan A. Dobrescu ${ }^{b}$ \\ ${ }^{a}$ Department of Physics, University of Wisconsin-Madison, \\ Madison, WI 53706, U.S.A. \\ ${ }^{b}$ Theoretical Physics Department, Fermilab, \\ Batavia, IL 60510, U.S.A. \\ E-mail: yangbai@physics.wisc.edu, bdob@fnal.gov
}

ABSTRACT: The coloron, a massive version of the gluon present in gauge extensions of QCD, has been searched for at the LHC as a dijet or top quark pair resonance. We point out that in the Renormalizable Coloron Model (ReCoM) with a minimal field content to break the gauge symmetry, a color-octet scalar and a singlet scalar are naturally lighter than the coloron because they are pseudo Nambu-Goldstone bosons. Consequently, the coloron may predominantly decay into scalar pairs, leading to novel signatures at the LHC. When the color-octet scalar is lighter than the singlet, or when the singlet mass is above roughly $1 \mathrm{TeV}$, the signatures consist of multi-jet resonances of multiplicity up to 12 , including topologies with multi-prong jet substructure, slightly displaced vertices, and sometimes a top quark pair. When the singlet is the lightest ReCoM boson and lighter than about $1 \mathrm{TeV}$, its main decays $\left(W^{+} W^{-}, \gamma Z, Z Z\right)$ arise at three loops. The LHC signatures then involve two or four boosted electroweak bosons, often originating from highly displaced vertices, plus one or two pairs of prompt jets or top quarks.

Keywords: Beyond Standard Model, Technicolor and Composite Models

ARXIV EPRINT: 1802.03005 


\section{Contents}

1 Introduction 1

2 Renormalizable SU(3) ${ }_{1} \times \mathrm{SU}(3)_{2}$ model 2

2.1 Bosonic mass spectrum 3

2.2 Interactions of the new bosons 5

$\begin{array}{ll}2.3 & \text { Partial widths of the color-octet bosons }\end{array}$

2.4 Partial widths of the singlet scalar $\phi_{I} \quad 8$

3 Production of scalars at the LHC 13

4 Novel LHC signatures $\quad \mathbf{1 5}$

4.1 Light color-octet scalar: $M_{G^{\prime}}>M_{\phi_{I}}>M_{\Theta} \quad 16$

$\begin{array}{lll}4.2 & \text { Light singlet scalar: } M_{G^{\prime}}>M_{\Theta}>M_{\phi_{I}} & 18\end{array}$

$\begin{array}{lll}4.2 .1 \quad \phi_{I} \rightarrow g g q \bar{q} & 19\end{array}$

4.2.2 $\phi_{I} \rightarrow W^{+} W^{-}, \gamma Z, Z Z \quad 23$

5 Discussion and conclusions $\quad 25$

$\begin{array}{ll}\text { A Ratios of scalar masses } & 29\end{array}$

B Coloron decays into scalars $\quad 31$

\section{Introduction}

Massive elementary particles of spin-1 are well-behaved at high-energies only if they are associated with a spontaneously broken gauge symmetry [1]. The sector responsible for breaking the gauge symmetry necessarily includes additional bosons (Higgs-like scalars or composite $\rho$-like fields) that couple to the gauge boson, and are not much heavier (but could be much lighter) than it. As a result, the additional bosons may drastically affect the phenomenology of a new massive gauge boson (see, e.g., $[2,3]$ ).

The coloron [4-7] is a color-octet spin-1 particle that is present in gauge extensions of the QCD. The classic example [8-10] is an $\mathrm{SU}(3)_{1} \times \mathrm{SU}(3)_{2}$ gauge symmetry broken down to the $\mathrm{SU}(3)_{c}$ gauge group of QCD. If all SM quarks are triplets under one of the $\mathrm{SU}(3)$ groups, then the coloron has flavor-universal couplings $[6,7]$, and dijet or top quark pair resonance searches at the LHC are sensitive to $s$-channel production of the coloron.

The spontaneous breaking of $\mathrm{SU}(3)_{1} \times \mathrm{SU}(3)_{2}$ is usually assumed to be due to a bifundamental scalar field that acquires a diagonal vacuum expectation value (VEV). A scalar potential that achieves that was considered in $[6,7]$, and involved a mass term and two quartic terms. It was pointed out in [2] that an additional term, of dimension 3 , in the potential is consistent with the gauge and Lorentz symmetries. The presence of that dimension-3 term is in fact useful, as it leads to a mass for a Nambu-Goldstone boson that 
would otherwise be phenomenologically problematic. The regions of parameter space where the desired symmetry breaking pattern is achieved have been derived in [11]. We refer to the flavor-universal $\mathrm{SU}(3)_{1} \times \mathrm{SU}(3)_{2}$ theory, with the most general terms of dimension up to four in the potential, as the Renormalizable Coloron Model (ReCoM).

Even though the ReCoM is a simple extension of the Standard Model (SM), its phenomenology is rich and has not been fully explored thus far. Besides the coloron, there are three physical spin-0 particles [2]: a color-octet, $\Theta$, and two singlets, $\phi_{I}$ and $\phi_{R}$. The constraints on the latter, which is a radial mode, have been derived in $[12,13]$ based on Higgs measurements, direct searches at the LHC, and electroweak observables. The processes discussed in ref. [2] rely on the existence of a vector-like quark, which is not part of the minimal structure of the ReCoM.

In this paper, we systematically study the novel collider signatures in the ReCoM and point out new search strategies for discovering the coloron and the associated scalars. The color-octet scalar and $\phi_{I}$ are pseudo-Nambu-Goldstone bosons (pNGB's), and thus are naturally lighter than the coloron. Depending on the scalar masses and on the amount of mixing of the $\mathrm{SU}(3)_{1} \times \mathrm{SU}(3)_{2}$ gauge bosons, the main decay channel of the coloron could be either $G_{\mu}^{\prime} \rightarrow \Theta \phi_{I}$ or $\Theta \Theta$, while the dijet branching fraction may be below $10 \%$.

The $\Theta$ and $\phi_{I}$ scalars have competing multi-body or multi-loop decays, as well as cascade decays in association with SM quark-antiquark pairs. As a result, the coloron appears as an $s$-channel multi-jet resonance, with two or more jet sub-clusters, and a total jet multiplicity as high as 12 when there are no top quarks produced. Channels that include $t \bar{t}$ pairs have even more complicated final states. For a lighter $\phi_{I}$, below around $1 \mathrm{TeV}$, its main decay channels are into two electroweak gauge bosons. The collider signatures in that case include boosted $W^{+} W^{-}, \gamma Z$ or $Z Z$ resonances, which typically originate from displaced vertices, plus a number of prompt quark jets or $t \bar{t}$ pairs. Currently, there are no dedicated searches at the LHC for these classes of signatures. The ReCoM thus provides a motivation for new searches at the LHC with complicated hadronic final states, various boosted systems, and displaced vertices.

The paper is organized as follows. We first work out the properties of the scalars in the ReCoM including their interactions and branching fractions in section 2. In section 3 , we calculate the production cross sections in phenomenologically relevant channels. In section 4, we identify the LHC signatures for different mass spectra and comment on search strategies. Our conclusions and two tables that summarize the main collider signatures are included in section 5. In appendix A, we derive the constraints on the scalar masses imposed by the condition that the color-conserving vacuum is the global minimum of the potential. In appendix B we outline the computation of the partial widths for coloron decays into scalars.

\section{Renormalizable $\mathrm{SU}(3)_{1} \times \mathrm{SU}(3)_{2}$ model}

We study a gauge extension of the $\mathrm{SM}$ with an $\mathrm{SU}(3)_{1} \times \mathrm{SU}(3)_{2} \times \mathrm{SU}(2)_{W} \times \mathrm{U}(1)_{Y}$ gauge group and a new scalar field $\Sigma$ transforming in the $(3, \overline{3}, 1,0)$ representation. The most 
general renormalizable potential that involves only $\Sigma$ has four terms:

$$
V(\Sigma)=-m_{\Sigma}^{2} \operatorname{Tr}\left(\Sigma \Sigma^{\dagger}\right)-\left(\mu_{\Sigma} \operatorname{det} \Sigma+\text { H.c. }\right)+\frac{\lambda}{2}\left[\operatorname{Tr}\left(\Sigma \Sigma^{\dagger}\right)\right]^{2}+\frac{\kappa}{2} \operatorname{Tr}\left(\Sigma \Sigma^{\dagger} \Sigma \Sigma^{\dagger}\right) .
$$

The squared-mass parameter $m_{\Sigma}^{2}$ can be positive or negative. The phase-rotation freedom of $\Sigma$ allows us to choose the mass parameter $\mu_{\Sigma}$ to be real and positive without loss of generality. At least one of the dimensionless couplings $\lambda$ and $\kappa$ must be positive. If one of them is negative, then the potential is bounded from below only when both $\lambda+\kappa$ and $3 \lambda+\kappa$ are positive [11].

At the renormalizable level, there is also a potential term for $\Sigma$ coupling to the SM Higgs doublet: $\operatorname{Tr}\left(\Sigma \Sigma^{\dagger}\right) H H^{\dagger}$, which modifies the Higgs boson properties [12, 13]. In the following, we will assume a small coupling for this interaction so that the modifications to the Higgs boson properties are below the current experimental sensitivity.

\subsection{Bosonic mass spectrum}

As studied in detail in ref. [11] (see also [14, 15]), and depending on the values of the four parameters in eq. (2.1), there are three possible vacua with the gauge symmetries given by the diagonal subgroup $\mathrm{SU}(3)_{c}$, or $\mathrm{SU}(2)_{1} \times \mathrm{SU}(2)_{2} \times \mathrm{U}(1)$, or the full $\mathrm{SU}(3)_{1} \times \mathrm{SU}(3)_{2}$. In ReCoM the phenomenologically viable regions of parameter space are those where the global minimum has the $\mathrm{SU}(3)_{c}$ symmetry, identified with the QCD gauge group. These regions of parameter space are summarized in appendix $\mathrm{A}$.

The $\Sigma$ field is a $3 \times 3$ matrix with complex entries, and its VEV is

$$
\langle\Sigma\rangle=\frac{f_{\Sigma}}{\sqrt{6}} \mathbb{I}_{3},
$$

with $\mathbb{I}_{3}$ the unit matrix, and the scale of $\mathrm{SU}(3)_{1} \times \mathrm{SU}(3)_{2}$ breaking given by

$$
f_{\Sigma}=\frac{\sqrt{3}}{\sqrt{2}(3 \lambda+\kappa)}\left(\sqrt{4(3 \lambda+\kappa) m_{\Sigma}^{2}+\mu_{\Sigma}^{2}}+\mu_{\Sigma}\right)>0 .
$$

Eight of the degrees of freedom in $\Sigma$ become the longitudinal degrees of freedom of the heavy gauge boson (the coloron), while the remaining ten degrees of freedom form a color-octet real scalar, $\Theta^{a}$, and two singlet real scalars, $\phi_{R}$ and $\phi_{I}$. The relation between these physical states and the uneaten degrees of freedom in $\Sigma$ is

$$
\Sigma=\frac{f_{\Sigma}+\phi_{R}+i \phi_{I}}{\sqrt{6}} \mathbb{I}_{3}+\Theta^{a} T^{a}
$$

where $T^{a}$ are the $\mathrm{SU}(3)_{c}$ generators, normalized as $\operatorname{Tr}\left(T^{a} T^{b}\right)=\frac{1}{2} \delta^{a b}$. Since the new scalars in the ReCoM do not have tree-level couplings to fermions, they are $\mathcal{P}$-even. Under charge conjugation the scalars transform as $\Sigma \stackrel{\mathcal{C}}{\rightarrow} \Sigma^{*}$, which implies $\Theta^{a} T^{a} \stackrel{\mathcal{C}}{\rightarrow} \Theta^{a}\left(T^{a}\right)^{*}, \phi_{R} \stackrel{\mathcal{C}}{\rightarrow} \phi_{R}$, and $\phi_{I} \stackrel{\mathcal{C}}{\rightarrow}-\phi_{I}$. Thus, $\phi_{I}$ is a $\mathcal{C} \mathcal{P}$-odd scalar which is also $\mathcal{C}$-odd. Its $\mathcal{C} \mathcal{P}$ property will determine its couplings to SM particles and lifetime, which will be discussed later. The 
squared-masses of the three scalar particles are given by

$$
\begin{aligned}
M_{\Theta}^{2} & =\frac{\kappa}{3} f_{\Sigma}^{2}+\sqrt{\frac{2}{3}} \mu_{\Sigma} f_{\Sigma}, \\
M_{\phi_{I}}^{2} & =\sqrt{\frac{3}{2}} \mu_{\Sigma} f_{\Sigma}, \\
M_{\phi_{R}}^{2} & =\left(\lambda+\frac{\kappa}{3}\right) f_{\Sigma}^{2}-\frac{\mu_{\Sigma}}{\sqrt{6}} f_{\Sigma} .
\end{aligned}
$$

Imposing that the $\mathrm{SU}(3)_{c}$-preserving vacuum is the global minimum of the potential, we prove in appendix $\mathrm{A}$ that there is an upper bound for the ratio of the $\phi_{I}$ and $\Theta$ masses: $M_{\phi_{I}} / M_{\Theta}<2.1$. In the limit of $\mu_{\Sigma} \ll f_{\Sigma}$, we have $M_{\phi_{I}} \ll M_{\phi_{R}}, M_{\Theta}$, indicating that the color-singlet scalar $\phi_{I}$ becomes the light pNGB associated with the spontaneous breaking of a global $\mathrm{U}(1)_{\Sigma}$ symmetry acting on $\Sigma$. The other color-singlet scalar $\phi_{R}$ has a mass of order $\sqrt{\lambda+\kappa / 3} f_{\Sigma}$, unless a fine-tuning of parameters makes it much lighter.

The limit of $\kappa \ll 1$ and $\mu_{\Sigma} \ll f_{\Sigma}$ is also interesting, as it enhances the global symmetry of the potential to $\mathrm{SO}(18)$, which is the symmetry that rotates the degrees of freedom of $\Sigma$. This symmetry is spontaneously broken down to $\mathrm{SO}(17)$ by $\langle\Sigma\rangle$, so that there are 17 light pNGB's in this case, which are the degrees of freedom within the longitudinal coloron, the color-octet scalar $\Theta$, and $\phi_{I}$. In that limit both $\Theta$ and $\phi_{I}$ are naturally lighter than the coloron and $\phi_{R}$.

Let us label the $\mathrm{SU}(3)_{1} \times \mathrm{SU}(3)_{2}$ gauge bosons by $G_{1}$ and $G_{2}$, and the corresponding gauge couplings by $h_{1}$ and $h_{2}$. One linear combination of the gauge bosons becomes the massless QCD gluon $(G)$ and the orthogonal combination is massive, the coloron $\left(G^{\prime}\right)$ :

$$
\begin{aligned}
G^{\mu} & =G_{1}^{\mu} \cos \theta+G_{2}^{\mu} \sin \theta, \\
G^{\prime \mu} & =G_{1}^{\mu} \sin \theta-G_{2}^{\mu} \cos \theta,
\end{aligned}
$$

where the mixing angle satisfies

$$
\tan \theta=\frac{h_{1}}{h_{2}} .
$$

The QCD gauge coupling is related to the $\mathrm{SU}(3)_{1} \times \mathrm{SU}(3)_{2}$ couplings by

$$
g_{s}=\frac{h_{1} h_{2}}{\sqrt{h_{1}^{2}+h_{2}^{2}}}
$$

and the coloron mass is

$$
M_{G^{\prime}}=\frac{g_{s}}{\sqrt{6}}\left(\tan \theta+\frac{1}{\tan \theta}\right) f_{\Sigma} .
$$

The gauge couplings are perturbative provided $3 h_{i}^{2} /\left(16 \pi^{2}\right) \lesssim 1$, based on naive dimensional analysis. These upper limits together with eq. (2.8) translate into a constraint on $\tan \theta: 0.15 \lesssim \tan \theta \lesssim 6.7$. We will use this range of $\tan \theta$ for our phenomenological analysis. We point out, however, that the tree-level analysis is more under control if the upper limit on the gauge couplings is lowered, e.g, $3 h_{i}^{2} /\left(16 \pi^{2}\right) \lesssim 1 / 3$, which gives $0.25 \lesssim \tan \theta \lesssim 4$. 


\subsection{Interactions of the new bosons}

The interactions among physical scalars include a trilinear $\Theta$ term,

$$
\bar{\mu}_{\Sigma} d^{a b c} \Theta^{a} \Theta^{b} \Theta^{c}
$$

where $d^{a b c}$ is the totally-symmetric color tensor. The coefficient $\bar{\mu}_{\Sigma}$ has mass dimension one, and receives contributions from the trilinear term in the $V(\Sigma)$ potential as well as from a quartic term in $V(\Sigma)$ with one insertion of the $\Sigma$ VEV:

$$
\begin{aligned}
\bar{\mu}_{\Sigma} & =\sqrt{\frac{3}{2}} \kappa f_{\Sigma}-\mu_{\Sigma} \\
& =\frac{3 g_{s}\left(1+\tan ^{2} \theta\right)}{2 M_{G^{\prime}} \tan \theta}\left(M_{\Theta}^{2}-\frac{8}{9} M_{\phi_{I}}^{2}\right),
\end{aligned}
$$

where in the second line we have used the relations between parameters and boson masses from eqs. (2.5) and (2.9). There are also trilinear interactions involving one $\phi_{R}$ singlet and either two color-octets or two $\phi_{I}$ singlets,

$$
\frac{1}{2}\left[(\lambda+\kappa) f_{\Sigma}+\frac{\mu_{\Sigma}}{\sqrt{6}}\right] \phi_{R} \Theta^{a} \Theta^{a}+\frac{1}{2}\left[\left(\lambda+\frac{\kappa}{3}\right) f_{\Sigma}+\frac{2 \mu_{\Sigma}}{\sqrt{6}}\right] \phi_{R} \phi_{I}^{2} .
$$

The only other trilinear scalar interaction involves three $\phi_{R}$ and is less phenomenologically important.

The interactions of a single coloron with scalars are given by

$$
\frac{g_{s}}{\tan \theta} G_{\mu}^{\prime a}\left[\frac{1}{\sqrt{6}}\left(1+\tan ^{2} \theta\right)\left(\phi_{I} \partial^{\mu} \Theta^{a}-\Theta^{a} \partial^{\mu} \phi_{I}\right)-\frac{1}{2}\left(1-\tan ^{2} \theta\right) f^{a b c} \Theta^{b} \partial^{\mu} \Theta^{c}\right],
$$

where $f^{a b c}$ is the totally-antisymmetric color tensor. Note that, for $\tan \theta=1$, the coupling of $G_{\mu}^{\prime}$ to two $\Theta$ 's vanishes. This is because when $h_{1}=h_{2}$ [see eq. (2.7)] there is an interchanging $\mathcal{Z}_{2}$ symmetry, under which $G_{1}^{\mu} \leftrightarrow G_{2}^{\mu}, G_{\mu} \leftrightarrow G_{\mu}, G_{\mu}^{\prime} \leftrightarrow-G_{\mu}^{\prime}, \Sigma \rightarrow \Sigma^{\dagger}$, $\phi_{I} \rightarrow-\phi_{I}$ and $\Theta^{a} \rightarrow \Theta^{a}$.

The couplings of a pair of colorons to a single scalar are

$$
\frac{M_{G^{\prime}}^{2}}{f_{\Sigma}}\left(\sqrt{\frac{3}{2}} d^{a b c} G_{\mu}^{\prime a} G^{\prime \mu b} \Theta^{c}+G_{\mu}^{\prime a} G^{\prime \mu a} \phi_{R}\right) .
$$

We will not display here the interactions of two colorons with two scalars, the quartic scalar interactions, or the coloron self-interactions (the latter are important if one of the $h_{i}$ gauge couplings is nearly nonperturbative).

For coloron interactions with fermions, we choose the simplest and anomaly-free model with the SM quarks as triplets under $\mathrm{SU}(3)_{1}$ and singlets under $\mathrm{SU}(3)_{2}$. The coloron interactions with quarks are flavor-blind and have the same form as those of the gluon, with an extra factor of $\tan \theta$ :

$$
g_{s} \tan \theta \bar{q} \gamma^{\mu} T^{a} G_{\mu}^{\prime a} q .
$$




\subsection{Partial widths of the color-octet bosons}

At tree-level and for $M_{\Theta}<M_{G^{\prime}}$, the main decay of the color-octet scalar is into two quark-antiquark pairs, via two off-shell colorons: $\Theta \rightarrow G^{* *} G^{*} \rightarrow q \bar{q} q^{\prime} \bar{q}^{\prime}$. Using eqs. (2.14) and (2.9), the 4-body width to leading order in $M_{\Theta}^{2} \ll M_{G^{\prime}}^{2}$ is parametrically of the order of

$$
\Gamma\left(\Theta \rightarrow q \bar{q} q^{\prime} \bar{q}^{\prime}\right) \sim \frac{\alpha_{s}^{3}}{\pi^{2}} \tan ^{2} \theta\left(1+\tan ^{2} \theta\right)^{2} \frac{M_{\Theta}^{7}}{M_{G^{\prime}}^{6}},
$$

where the width is summed over the quark flavors, and $\alpha_{s}$ is the QCD coupling constant. We have assumed here that the phase-space suppression is of the order of $(4 \pi)^{5}$. For $M_{\Theta} \sim 1 \mathrm{TeV}, M_{G^{\prime}} \sim 4 \mathrm{TeV}$ and $\tan \theta \sim 0.3$, the 4-body width is at the keV scale. For larger $M_{\Theta}$, of order $M_{G^{\prime}} / 2, \Gamma\left(\Theta \rightarrow q \bar{q} q^{\prime} \bar{q}^{\prime}\right)$ is larger by a few orders of magnitude.

At one loop, $\Theta$ decays into two gluons. The amplitude for this process gets two contributions. The first one is from diagrams with $\Theta$ running in the loop and a vertex involving the trilinear interaction (2.10). The second contribution is from diagrams with the coloron running in the loop and the $G^{\prime} G^{\prime} \Theta$ vertex given in eq. (2.14). Adding the two contributions, we have the following effective interaction:

$$
\frac{C_{\Theta}}{f_{\Sigma}}\left(\frac{g_{s}^{2}}{16 \pi^{2}}\right) d^{a b c} \Theta^{a} G_{\alpha \beta}^{b} G^{c \alpha \beta},
$$

where the dimensionless coefficient is

$$
C_{\Theta}=6 \sqrt{2}\left(\frac{\pi^{2}}{9}-1\right) \frac{\bar{\mu}_{\Sigma} f_{\Sigma}}{M_{\Theta}^{2}}+\frac{3 \sqrt{6}}{32} \mathcal{A}\left[M_{\Theta}^{2} /\left(4 M_{G^{\prime}}^{2}\right)\right] .
$$

Here, the function $\mathcal{A}(\tau)=\left[2 \tau^{2}+3 \tau+3(2 \tau-1) \arcsin ^{2} \sqrt{\tau}\right] \tau^{-2}$ for $\tau \leq 1$, which is similar to the $W^{ \pm}$loop contributions to the effective interaction of the Higgs boson with two photons. In the $\tau \rightarrow 0$ limit, the function $\mathcal{A}(\tau)$ reaches its minimum, $\mathcal{A}\left(0_{+}\right) \rightarrow 7 ; \mathcal{A}(\tau)$ increases monotonically with $\tau$, and $A(1 / 4) \approx 7.42$. The ratio of the second term to the first term in eq. (2.18) has a simple formula:

$$
\begin{aligned}
r_{\mathcal{A}} & =\frac{3}{32 \sqrt{2}\left(\pi^{2}-9\right)} \frac{\mathcal{A}\left[M_{\Theta}^{2} /\left(4 M_{G^{\prime}}^{2}\right)\right]}{1-8 M_{\phi_{I}}^{2} /\left(9 M_{\Theta}^{2}\right)} \\
& \approx 0.533\left(1+\frac{8 M_{\phi_{I}}^{2}}{9 M_{\Theta}^{2}}+\frac{11 M_{\Theta}^{2}}{210 M_{G^{\prime}}^{2}}+\cdots\right),
\end{aligned}
$$

where the ellipsis in the second line refers to higher-order terms in $M_{\phi_{I}}^{2} / M_{\Theta}^{2}$ and $M_{\Theta}^{2} / M_{G^{\prime}}^{2}$. The above expression shows that the two contributions to $\Theta \rightarrow g g$ are comparable. Using the result for the scalar contribution given in [2], and including the interference with the coloron contribution, we find the $\Theta \rightarrow g g$ width:

$$
\begin{aligned}
\Gamma(\Theta \rightarrow g g) & =\frac{15 \alpha_{s}^{2} \bar{\mu}_{\Sigma}^{2}}{128 \pi^{3} M_{\Theta}}\left(\frac{\pi^{2}}{9}-1\right)^{2}\left(1+r_{\mathcal{A}}\right)^{2} \\
& \approx \frac{135 \alpha_{s}^{2}}{256 \pi^{3}}\left(\frac{\pi^{2}}{9}-1\right)^{2}\left(1+r_{\mathcal{A}}\right)^{2} \kappa M_{\Theta},
\end{aligned}
$$



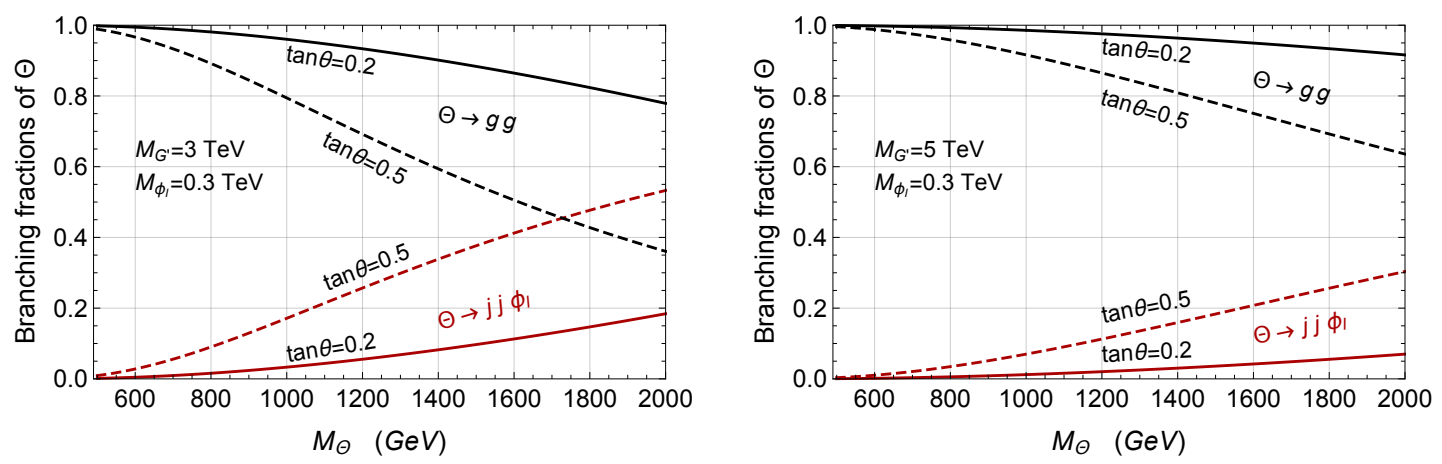

Figure 1. Branching fractions of the color-octet scalar $\Theta$ as a function of its mass. For $\Theta \rightarrow j j \phi_{I}$ the width is summed over the five light quark flavors in the final state. The branching fraction for $\Theta \rightarrow t \bar{t} \phi_{I}$ (not shown) is slightly less than $1 / 5$ of that for $\Theta \rightarrow j j \phi_{I}$. The coloron mass used here is $M_{G^{\prime}}=3 \mathrm{TeV}$ (left panel) or $5 \mathrm{TeV}$ (right panel), and the neutral scalar mass is fixed at $M_{\phi_{I}}=300 \mathrm{GeV}$. The parameter $\tan \theta$, which determines the strength of the coloron couplings, is taken to be 0.2 (solid lines) or 0.5 (dashed lines).

where the approximation in the second line is taken in the limit of $\mu_{\Sigma} \ll \kappa f_{\Sigma}$ (which implies $\left.M_{\phi_{I}} \ll M_{\Theta}\right)$. Note the accidental suppression of the above width by a factor of $\left(\pi^{2} / 9-1\right)^{2} \approx 9.3 \times 10^{-3}$. The interference of the scalar and coloron contributions is constructive for $M_{\phi_{I}}<6 \sqrt{2} M_{\Theta}$, which is automatically satisfied in the ReCoM (see appendix A). For $M_{\Theta}=1 \mathrm{TeV}, M_{G^{\prime}}=4 \mathrm{TeV}, M_{\phi_{I}}=300 \mathrm{GeV}$ and $\tan \theta \sim 0.3$, the 1-loop 2-body width of $\Theta$ is around $2 \mathrm{MeV}$, much above the tree-level 4-body one.

We also note that there is an effective interaction of $\Theta^{a}$ with two quarks, which can be generated at one loop, with a $G^{\prime}$ and a quark running in the loop. However, this scalarfermion interaction is suppressed by the ratio of fermion mass over coloron mass. Even for the top quark, the estimated width is smaller by two orders of magnitude than the one of $\Theta \rightarrow g g$. We will ignore this small branching fraction in our analysis.

If $M_{\Theta}>M_{\phi_{I}}$, then $\Theta$ also has 3-body tree-level decays into a $\phi_{I}$ scalar and a quarkantiquark pair of same flavor, $\Theta \rightarrow \bar{q} q \phi_{I}$, through an off-shell $G^{\prime}$. Neglecting the quark masses, the partial widths of $\Theta$ into a $\phi_{I}$ and either a pair of quark jets $(j j)$ or $t \bar{t}$ satisfy

$\Gamma\left(\Theta \rightarrow j j \phi_{I}\right) \simeq 5 \Gamma\left(\Theta \rightarrow \bar{t} t \phi_{I}\right) \simeq \frac{5 \alpha_{s}^{2} M_{\Theta}^{5}}{576 \pi M_{G^{\prime}}^{4}}\left(1+\tan ^{2} \theta\right)^{2} \mathcal{F}\left(M_{\Theta}^{2} / M_{G^{\prime}}^{2}, M_{\phi_{I}}^{2} / M_{G^{\prime}}^{2}\right)$,

where we defined the function

$$
\begin{aligned}
\mathcal{F}(x, y)=\frac{12}{x^{4}} & {\left[(y-x)\left(1-\frac{3}{2}(x+y)+\frac{1}{3}(x-y)^{2}\right)+\frac{1}{2}\left(1-2(x+y)+x^{2}+y^{2}\right) \log \left(\frac{x}{y}\right)\right.} \\
& \left.+(1-x-y)\left(1-2(x+y)+(x-y)^{2}\right)^{1 / 2} \tanh ^{-1}\left(\frac{\left(1-2(x+y)+(x-y)^{2}\right)^{1 / 2}}{x-y-(x+y) /(x-y)}\right)\right] .
\end{aligned}
$$

In figure 1, we show the branching fractions of 2- and 3-body decays as a function of $M_{\Theta}$ for fixed masses of $\phi_{I}$ and the coloron. Note that the 3-body branching fraction increases 

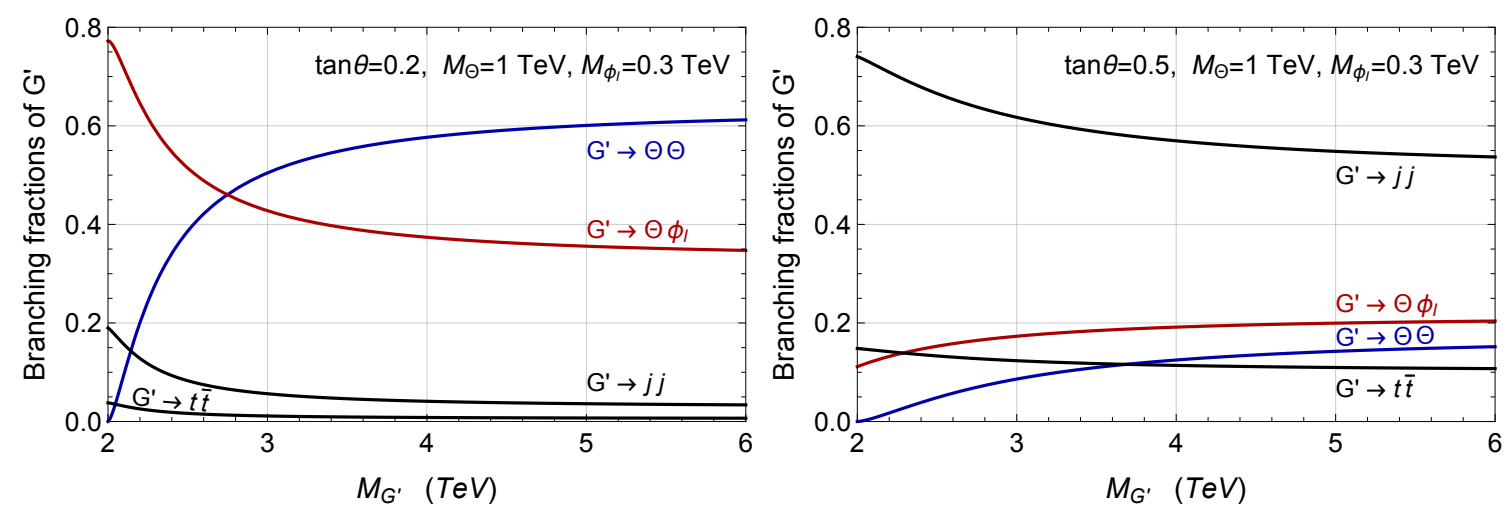

Figure 2. Branching fractions of the coloron, $G^{\prime}$, as a function of its mass, for $\tan \theta=0.2$ (left panel) or $\tan \theta=0.5$ (right panel). The scalar masses are fixed at $M_{\Theta}=1 \mathrm{TeV}$ and $M_{\phi_{I}}=300 \mathrm{GeV}$.

as $M_{\Theta}$ increases. For a fixed value of $M_{\Theta}$, increasing the mixing parameter $\tan \theta$ leads to an increase of the 3-body branching fraction. It is helpful to take the $M_{\Theta}, M_{\phi_{I}} \ll M_{G^{\prime}}$ limit (for any $M_{\Theta}>M_{\phi_{I}}$ ) in eq. (2.21), which corresponds to the following limit:

$$
\mathcal{F}(x, y) \underset{x, y \ll 1}{\longrightarrow} 1-8 \frac{y}{x}+12 \frac{y^{2}}{x^{2}} \log \left(\frac{x}{y}\right)+8 \frac{y^{3}}{x^{3}}-\frac{y^{4}}{x^{4}} .
$$

The coloron with a mass above the scalar pair thresholds, $\left(M_{G^{\prime}}>M_{\Theta}+M_{\phi_{I}}, 2 M_{\Theta}\right)$ has four 2-body decay channels with tree-level widths:

$$
\begin{aligned}
\Gamma\left(G_{\mu}^{\prime} \rightarrow \Theta \phi_{I}\right) & =\frac{\alpha_{s}}{72 \tan ^{2} \theta}\left(1+\tan ^{2} \theta\right)^{2} M_{G^{\prime}}\left(1-2 \frac{M_{\Theta}^{2}+M_{\phi_{I}}^{2}}{M_{G^{\prime}}^{2}}+\frac{\left(M_{\Theta}^{2}-M_{\phi_{I}}^{2}\right)^{2}}{M_{G^{\prime}}^{4}}\right)^{3 / 2}, \\
\Gamma\left(G_{\mu}^{\prime} \rightarrow \Theta \Theta\right) & =\frac{\alpha_{s}}{32 \tan ^{2} \theta}\left(1-\tan ^{2} \theta\right)^{2} M_{G^{\prime}}\left(1-\frac{4 M_{\Theta}^{2}}{M_{G^{\prime}}^{2}}\right)^{3 / 2}, \\
\Gamma\left(G_{\mu}^{\prime} \rightarrow j j\right) & =\frac{5 \alpha_{s}}{6} \tan ^{2} \theta M_{G^{\prime}}, \\
\Gamma\left(G_{\mu}^{\prime} \rightarrow t \bar{t}\right) & =\frac{\alpha_{s}}{6} \tan ^{2} \theta M_{G^{\prime}}\left(1-\frac{4 m_{t}^{2}}{M_{G^{\prime}}^{2}}\right)^{1 / 2} .
\end{aligned}
$$

The computation of the partial widths for coloron decays into scalars (first two equations above) is outlined in appendix B. The partial width into a pair of quark jets $(j j)$ is summed over five flavors. In figure 2, we show the branching fractions of $G^{\prime}$ as a function of $M_{G^{\prime}}$. For $\tan \theta$ larger than about 0.45 (as in the right-hand panel), the decays of $G^{\prime}$ are dominated by the dijet channel, while for a smaller value of $\tan \theta$ (as in the left-hand panel), the decays into $\Theta \phi_{I}$ and $\Theta \Theta$ have large branching fractions.

\subsection{Partial widths of the singlet scalar $\phi_{I}$}

The main decay channel of the $\phi_{I}$ scalar, in the case of the $M_{\phi_{I}}>M_{\Theta}$ mass ordering, is a 3-body one: $\phi_{I} \rightarrow j j \Theta$ via an off-shell $G^{\prime}$ with a width

$\Gamma\left(\phi_{I} \rightarrow j j \Theta\right) \simeq 5 \Gamma\left(\phi_{I} \rightarrow \bar{t} t \Theta\right) \approx \frac{5 \alpha_{s}^{2} M_{\phi_{I}}^{5}}{72 \pi M_{G^{\prime}}^{4}}\left(1+\tan ^{2} \theta\right)^{2} \mathcal{F}\left(M_{\phi_{I}}^{2} / M_{G^{\prime}}^{2}, M_{\Theta}^{2} / M_{G^{\prime}}^{2}\right)$, 

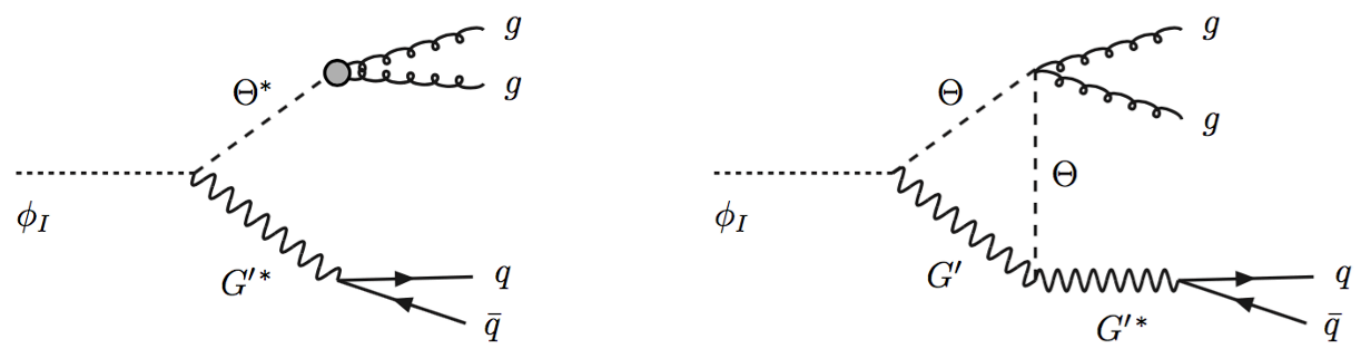

Figure 3. Diagrams responsible for the 4-body decay $\phi_{I} \rightarrow g g q \bar{q}$ of the $\mathcal{C} \mathcal{P}$-odd scalar when $M_{\phi_{I}}<M_{\Theta}$. Left diagram: decay through an off-shell coloron and an off-shell $\Theta$ scalar, which couples at one loop to two gluons. Right diagram: 1-loop decay into a gluon pair and an off-shell coloron (other ways of attaching the gluons to the loop are not shown). The coloron interactions with scalars are given in eq. (2.13).

where the function $\mathcal{F}(x, y)$ is defined in eq. (2.22). Comparing eqs. (2.21) and (2.25), note that the order of $M_{\Theta}$ and $M_{\phi_{I}}$ is reversed in the arguments of $\mathcal{F}$.

In the case of the other mass ordering, $M_{\phi_{I}}<M_{\Theta}$, the leading decay of $\phi_{I}$ is likely to be a 4-body one into two gluons plus two quarks via the diagrams shown in figure 3 . The first diagram there is the decay via an off-shell $G^{\prime}$ and an off-shell $\Theta$. Note that the $\Theta$ coupling to a gluon pair arises at one loop, and is described by the dimension- 5 operator (2.17), with the coefficient $C_{\Theta}$ defined in eq. (2.18). Extracting $f_{\Sigma}$ from eq. (2.9), and using the expressions for $\bar{\mu}_{\Sigma}$ and $r_{\mathcal{A}}$ given in eqs. (2.11) and (2.19), we find

$$
C_{\Theta} \approx 4.61\left(1-0.58 \frac{M_{\phi_{I}}^{2}}{M_{\Theta}^{2}}+0.018 \frac{M_{\Theta}^{2}}{M_{G^{\prime}}^{2}}+\cdots\right),
$$

where higher-order terms in $M_{\phi_{I}}^{2} / M_{\Theta}^{2}$ and $M_{\Theta}^{2} / M_{G^{\prime}}^{2}$ are represented by the dots. The effective coupling of $\phi_{I}$ to gluons and quarks induced by the first diagram in figure 3 is described in the $M_{\phi_{I}}^{2} \ll M_{\Theta}^{2}, M_{G^{\prime}}^{2}$ limit by a dimension-9 operator:

$$
C_{\Theta} \frac{g_{s}^{3} \tan \theta}{32 \pi^{2} f_{\Sigma}^{2} M_{G^{\prime}} M_{\Theta}^{2}} d^{a b c}\left(\partial_{\mu} \phi_{I}\right) \bar{q} \gamma^{\mu} T^{a} q G_{\alpha \beta}^{b} G^{c \alpha \beta} .
$$

The second diagram shown in figure 3 , together with four similar 1-loop diagrams where the two gluons are attached in different ways to the $\Theta$ and $G^{\prime}$ internal lines, contribute to the same operator (for $M_{\phi_{I}}^{2} \ll M_{\Theta}^{2}$ ), as well as to other dimension-9 operators with different Lorentz contractions. All these contributions are parametrically of the same order. Assuming that $\phi_{I}$ is much heavier than the QCD scale (so we do not need to take into account hadronization effects), the 4-body width of $\phi_{I}$ summed over 5 light quark flavors can be written as

$$
\begin{aligned}
\Gamma\left(\phi_{I} \rightarrow g g q \bar{q}\right) & =\frac{2}{189(4 \pi)^{5}}\left(C_{\Theta}+C_{\Theta}^{\prime}\right)^{2}\left(\frac{g_{s}^{3} \tan \theta}{32 \pi^{2} f_{\Sigma}^{2} M_{G^{\prime}} M_{\Theta}^{2}}\right)^{2} M_{\phi_{I}}^{11}, \\
& =\frac{\alpha_{s}^{5}}{168(12 \pi)^{4}}\left(C_{\Theta}+C_{\Theta}^{\prime}\right)^{2} \frac{\left(1+\tan ^{2} \theta\right)^{4}}{\tan ^{2} \theta} \frac{M_{\phi_{I}}^{11}}{M_{\Theta}^{4} M_{G^{\prime}}^{6}},
\end{aligned}
$$


where $C_{\Theta}^{\prime}$ is a function of $M_{\Theta} / M_{G^{\prime}}$ that takes into account the 1-loop diagrams of the type shown on the right-hand side of figure 3 . We expect that $C_{\Theta}^{\prime}$ is typically of order one or smaller. There is also a $\phi_{I} \rightarrow g g t \bar{t}$ decay, whose width is smaller by a factor of 5 (or more when $M_{\phi_{I}}$ is not much larger than $2 m_{t}$ ). For $M_{\phi_{I}}=700 \mathrm{GeV}, M_{\Theta}=1 \mathrm{TeV}, M_{G^{\prime}}=3 \mathrm{TeV}$ and $\tan \theta=0.3$, the width of $\phi_{I}$ is of the order of $5 \times 10^{-13} \mathrm{GeV}$, corresponding to a lifetime $\tau_{\phi_{I}}^{0} \approx 10^{-12}$ s. Note that the lifetime is proportional to $M_{\phi_{I}}^{-11}$. Thus, a lighter $\phi_{I}$ produced at the LHC can easily have a long displaced vertex if the 4-body decay is the dominant channel. In section 4.2 we will estimate the $\phi_{I}$ decay length in the lab frame.

One may wonder about 2-body decay channels like $\phi_{I} \rightarrow g g$, as in the case of $\Theta$ discussed earlier (see section 2.3). As pointed out below eq. (2.4), the $\phi_{I}$ is a $\mathcal{C}$-odd and $\mathcal{P}$-even scalar. Its decay into two gluons is therefore highly suppressed by chargeconjugation symmetry, requiring electroweak interactions, which enter only at four loops. That symmetry will not forbid $\phi_{I}$ decays into three gluons. The lowest-dimension operator that is $\mathcal{C}$-conserving appears to be $\phi_{I} d^{a b c} G_{\nu}^{a \mu} G_{\mu}^{b \alpha} G_{\alpha}^{c \nu}$, but this operator vanishes because the gluon field strength is antisymmetric in Lorentz indices. At the same dimension, there exist two more operators, $\phi_{I} f^{a b c} G_{\nu}^{a \mu} G_{\mu}^{b \alpha} G_{\alpha}^{c \nu}$ and $\phi_{I} f^{a b c} G_{\nu}^{a \mu} G_{\mu}^{b \alpha} \widetilde{G}_{\alpha}^{c \nu}$, both of which are $\mathcal{C}$-violating and thus highly suppressed, again being generated only at four loops. ${ }^{1}$ The next operators are at dimension 9, including $d^{a b c}\left(\partial_{\mu} \phi_{I}\right)\left(D_{\nu} G^{a \mu \nu}\right) G_{\alpha \beta}^{b} G^{c \alpha \beta}$, and have the same structure as the one in eq. (2.27) after using the field equation for the gluon.

Another possible decay of $\phi_{I}$ is into a quark-antiquark pair, with or without one more boson. To conserve $\mathcal{C}$, the effective operator below electroweak symmetry breaking is $\left(\partial_{\mu} \phi_{I}\right) \bar{t} \gamma^{\mu} t$, which is zero after using the fermion equation of motion. The next nonvanishing operator is at dimension $7,\left(\partial_{\mu} \phi_{I}\right) H H^{\dagger} \bar{t} \gamma^{\mu} t$, where $H$ is the Higgs doublet. This includes the non-vanishing interaction $\phi_{I}\left(\partial_{\mu} h^{0}\right) \bar{t} \gamma^{\mu} t$, where $h^{0}$ is the SM Higgs boson. This interaction can be generated by a 2-loop diagram (see the left panel of figure 4), with $\Theta$ and $G^{\prime}$ running in the loop, and can be written as

$$
\eta_{1}\left(M_{\Theta} / M_{G^{\prime}}\right) \tan \theta\left(1+\tan ^{2} \theta\right)^{2} \frac{10 \sqrt{2} g_{s}^{5} y_{t}^{2} v_{\mathrm{EW}}}{9\left(16 \pi^{2}\right)^{2} M_{G^{\prime}}^{3}} \phi_{I}\left(\partial_{\mu} h^{0}\right) \bar{t} \gamma^{\mu} t
$$

Here, $v_{\mathrm{EW}} \approx 246 \mathrm{GeV}$ is the Higgs VEV; $y_{t}$ is the top quark Yukawa coupling; $\eta_{1}$ is a dimensionless coefficient which we expect to be of order one, or slightly smaller; the factor $10 / 9$ comes from color contractions. For $M_{\phi_{I}}>m_{h}+2 m_{t} \approx 470 \mathrm{GeV}$ and ignoring the final state phase-space factor, the 3-body decay width is calculated to be

$$
\Gamma\left(\phi_{I} \rightarrow h^{0} t \bar{t}\right) \simeq \frac{25 \alpha_{s}^{5} \eta_{1}^{2} y_{t}^{4}}{162(4 \pi)^{6}} \tan ^{2} \theta\left(1+\tan ^{2} \theta\right)^{4} \frac{v_{\mathrm{EW}}^{2} M_{\phi_{I}}^{5}}{M_{G^{\prime}}^{6}} .
$$

The decays into lighter quarks are further suppressed by small Yukawa couplings and are negligible. Combining (2.28) and (2.30), the ratio of the 2-loop 3-body and 1-loop 4-body decays of $\phi_{I}$ is

$$
\frac{\Gamma\left(\phi_{I} \rightarrow h^{0} t \bar{t}\right)}{\Gamma\left(\phi_{I} \rightarrow g g q \bar{q}\right)} \approx 2.6 \times 10^{-3} \eta_{1}^{2}\left(\frac{\tan \theta}{0.3}\right)^{4}\left(\frac{M_{\Theta}}{1 \mathrm{TeV}}\right)^{4}\left(\frac{700 \mathrm{GeV}}{M_{\phi_{I}}}\right)^{6} .
$$

\footnotetext{
${ }^{1}$ The $\mathcal{C}$ - and $\mathcal{C} \mathcal{P}$-conserving dimension-6 gluon operators without the $\mathcal{C}$-odd $\phi_{I}$ field were studied in refs. $[16,17]$.
} 

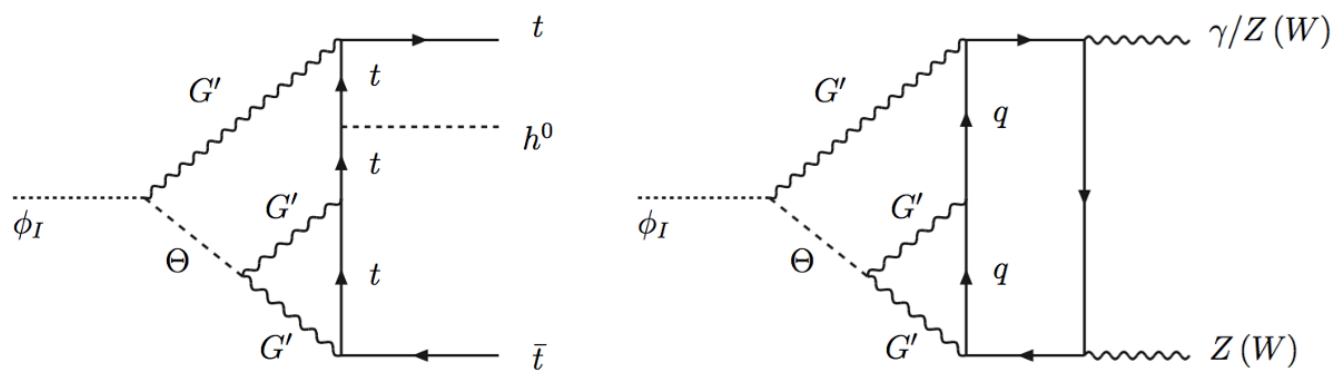

Figure 4. Additional decays of the $\mathcal{C P}$-odd scalar when $M_{\phi_{I}}<M_{\Theta}$. Left diagram: $\phi_{I} \rightarrow t \bar{t} h^{0}$ at two loops. Right diagram: $\phi_{I} \rightarrow \gamma Z, Z Z, W^{+} W^{-}$at three loops.

Hence, the 2-loop 3-body decay could be relevant only for a small $M_{\phi_{I}}$, below around $300 \mathrm{GeV}$, in which case the collider signature involves a displaced vertex.

As both $\mathcal{C}$ and $\mathcal{P}$ are explicitly broken by the electroweak interactions, there are more $\mathcal{C}$ and $\mathcal{P}$-violating operators for $\phi_{I}$ decaying into SM particles. At the 2-loop level, a Feynman diagram similar to the one on the left side of figure 4, with $h^{0}$ replaced by the hypercharge gauge boson $B_{\mu}$, generates the following representative dimension-7 operator:

$$
\eta_{2}\left(M_{\Theta} / M_{G^{\prime}}\right) \tan \theta\left(1+\tan ^{2} \theta\right)^{2} \frac{5 g_{s}^{5} e}{9\left(16 \pi^{2}\right)^{2} c_{W} M_{G^{\prime}}^{3}}\left(\partial^{\mu} \phi_{I}\right) \bar{q} \gamma^{\nu} \gamma_{5} q B_{\mu \nu}
$$

where $\eta_{2}$ is a coefficient of order one or smaller, and $c_{W}$ is the cosine of the weak mixing angle. The ensuing 2-loop 3-body decays, involving a photon or a $Z$ boson, have a width

$$
\Gamma\left(\phi_{I} \rightarrow \gamma q \bar{q}\right) \simeq \frac{c_{W}^{2}}{s_{W}^{2}} \Gamma\left(\phi_{I} \rightarrow Z q \bar{q}\right) \simeq \frac{5 \alpha \alpha_{s}^{5} \eta_{2}^{2}}{648(4 \pi)^{5}} \tan ^{2} \theta\left(1+\tan ^{2} \theta\right)^{4} \frac{M_{\phi_{I}}^{7}}{M_{G^{\prime}}^{6}}
$$

where we ignored the final state particle masses, and summed over 5 quark flavors. The ratio of these 2-loop 3-body and the 1-loop 4-body widths of $\phi_{I}$ is

$$
\frac{\Gamma\left(\phi_{I} \rightarrow \gamma / Z q \bar{q}\right)}{\Gamma\left(\phi_{I} \rightarrow g g q \bar{q}\right)} \approx 1.3 \times 10^{-4} \eta_{2}^{2}\left(\frac{\tan \theta}{0.3}\right)^{4}\left(\frac{M_{\Theta}}{1 \mathrm{TeV}}\right)^{4}\left(\frac{700 \mathrm{GeV}}{M_{\phi_{I}}}\right)^{4} .
$$

There are also decay channels involving the $W$ boson, $\phi_{I} \rightarrow W^{ \pm} q \bar{q}^{\prime}$ with comparable branching fractions. Thus, the 2-loop 3-body widths with an electroweak boson in the final state are probably too small to be relevant for collider studies.

At the 3-loop level, $\phi_{I}$ couples to two electroweak bosons or two SM fermions. A diagram of this type is shown on the right-hand side of figure 4 . The $\mathcal{C P}$-conserving operators that couple the $\mathcal{C P}$-odd scalar to two electroweak gauge bosons include

$\eta_{3}\left(M_{\Theta} / M_{G^{\prime}}\right) \tan \theta\left(1+\tan ^{2} \theta\right)^{2} \frac{5 g_{s}^{5} e^{2}}{2\left(16 \pi^{2}\right)^{3} M_{G^{\prime}}} \phi_{I}\left(\frac{1}{s_{W}^{2}} W_{\mu \nu}^{i} \widetilde{W}^{i \mu \nu}-\frac{1}{c_{W}^{2}} B_{\mu \nu} \widetilde{B}^{\mu \nu}\right)$,

where $\eta_{3}$ again is a coefficient of order one or smaller, which depends on the $\phi_{I}, \Theta$ and $G^{\prime}$ masses, and can be found by computing the 3-loops diagrams. The coefficient of the above operator for the hypercharge gauge boson includes a factor of $-3 / 2$, which accounts for 
the sum over the squared hypercharges of the six quark flavors running in the loop. The coefficient for the $W^{i}$ gauge bosons comes from three generations of quarks and $1 / 2$ for the normalization of $\mathrm{SU}(2)_{W}$ generators. The overall coefficient in (2.35) includes a color factor of $10 / 3$. Interestingly, after rotating the basis to $\gamma, Z$ and $W^{ \pm}$, there is no 3-loop coupling of $\phi_{I}$ to two photons. For other combinations of two gauge bosons, the 3-loop 2-body decays have widths

$$
\begin{aligned}
\Gamma\left(\phi_{I} \rightarrow \gamma Z\right) & \simeq \frac{s_{W}^{2}}{c_{W}^{2}} \Gamma\left(\phi_{I} \rightarrow W^{+} W^{-}\right) \simeq \frac{2 s_{W}^{2} c_{W}^{2}}{1-4 s_{W}^{2} c_{W}^{2}} \Gamma\left(\phi_{I} \rightarrow Z Z\right) \\
& \simeq \frac{25 \alpha^{2} \alpha_{s}^{5} \eta_{3}^{2}}{2(4 \pi)^{6} s_{W}^{2} c_{W}^{2}} \tan ^{2} \theta\left(1+\tan ^{2} \theta\right)^{4} \frac{M_{\phi_{I}}^{3}}{M_{G^{\prime}}^{2}} .
\end{aligned}
$$

The ratios of these 3 -loop 2-body widths to the 1-loop 4-body width of $\phi_{I}$ given in eq. (2.28) are

$$
\frac{\Gamma\left(\phi_{I} \rightarrow \gamma Z, W W, Z Z\right)}{\Gamma\left(\phi_{I} \rightarrow g g q \bar{q}\right)} \approx(0.20,0.66,0.16) \eta_{3}^{2}\left(\frac{\tan \theta}{0.3}\right)^{4}\left(\frac{M_{G^{\prime}}}{3 \mathrm{TeV}}\right)^{4}\left(\frac{M_{\Theta}}{1 \mathrm{TeV}}\right)^{4}\left(\frac{700 \mathrm{GeV}}{M_{\phi_{I}}}\right)^{8}
$$

If $\eta_{3}=O(1)$, the 2-body decays into two electroweak bosons become the leading decay channels of $\phi_{I}$ for $M_{\phi_{I}}$ below around $700 \mathrm{GeV}$, or for $M_{G^{\prime}} M_{\Theta}$ above $3 \mathrm{TeV}^{2}$. If $\eta_{3}$ turns out to be much smaller, $\eta_{3}^{2}=O\left(10^{-3}\right)$, then the 2-body decays become dominant for $M_{\phi_{I}} \lesssim 300 \mathrm{GeV}$ when $M_{G^{\prime}} M_{\Theta} \sim 3 \mathrm{TeV}^{2}$.

At the 3-loop level (e.g., the left diagram of figure 4 with an additional $W$ loop attached to the top quark lines and no $\left.h^{0}\right)$, the $\mathcal{C P}$-conserving couplings to two fermions contain the dimension-5 operator

$$
\eta_{4}\left(M_{\Theta} / M_{G^{\prime}}\right) \tan \theta\left(1+\tan ^{2} \theta\right)^{2} \frac{5 g_{s}^{5} e^{2}}{9\left(16 \pi^{2}\right)^{3} s_{W}^{2} M_{G^{\prime}}}\left(\partial_{\mu} \phi_{I}\right) \bar{t} \gamma^{\mu} \gamma_{5} t
$$

After using integration by parts and fermion equation of motion, it is easy to show that the coupling is proportional to the fermion mass, so the top quark (if kinematically allowed) is the most important channel. Ignoring the final-state fermion masses, this 3-loop 2-body decay width is then

$$
\Gamma\left(\phi_{I} \rightarrow t \bar{t}\right) \simeq \frac{25 \alpha^{2} \alpha_{s}^{5} \eta_{4}^{2}}{81(4 \pi)^{6} s_{W}^{4}} \tan ^{2} \theta\left(1+\tan ^{2} \theta\right)^{4} \frac{m_{t}^{2} M_{\phi_{I}}}{M_{G^{\prime}}^{2}} .
$$

The ratio of this decay mode over the 1-loop 4-body one is

$$
\frac{\Gamma\left(\phi_{I} \rightarrow t \bar{t}\right)}{\Gamma\left(\phi_{I} \rightarrow g g q \bar{q}\right)} \approx 1.0 \times 10^{-3} \eta_{4}^{2}\left(\frac{\tan \theta}{0.3}\right)^{4}\left(\frac{M_{G^{\prime}}}{3 \mathrm{TeV}}\right)^{4}\left(\frac{M_{\Theta}}{1 \mathrm{TeV}}\right)^{4}\left(\frac{700 \mathrm{GeV}}{M_{\phi_{I}}}\right)^{10}
$$

Comparing the two 3-loop widths in (2.36) and (2.39), the fermion pair channel is suppressed by $m_{t}^{2} / M_{\phi_{I}}^{2}$ for comparable $\eta_{3}$ and $\eta_{4}$.

In conclusion, for $M_{\phi_{I}}$ not dramatically below $M_{G^{\prime}}$ and $M_{\Theta}$, the main decay of $\phi_{I}$ is the 1-loop 4-body channel $\phi_{I} \rightarrow g g q \bar{q}$ in (2.28), otherwise the main channels appear to be $\phi_{I} \rightarrow \gamma Z, W^{+} W^{-}, Z Z$ with the widths estimated in (2.36). 


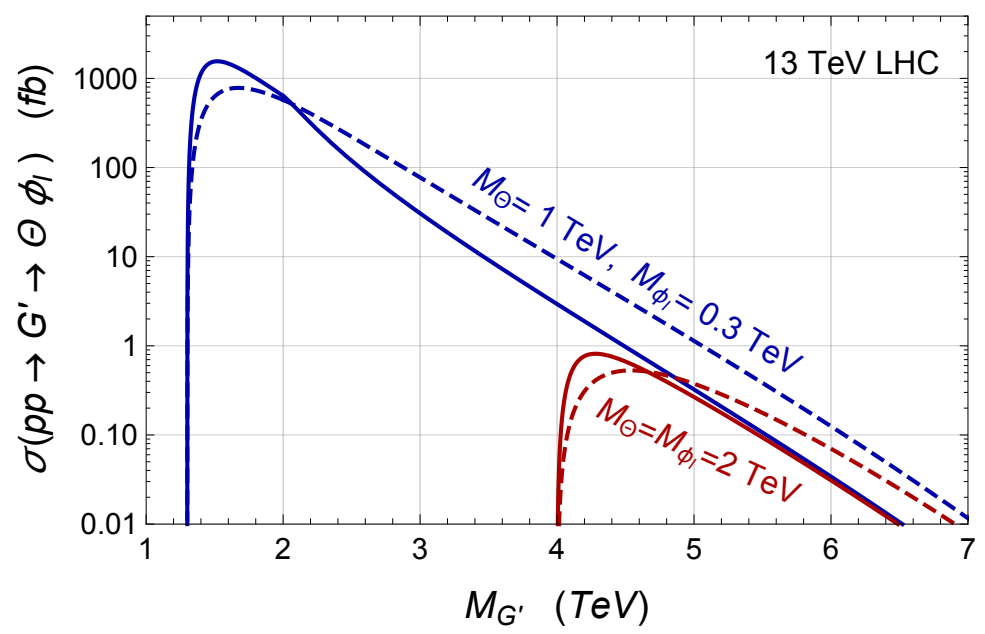

Figure 5. Cross section for the $p p \rightarrow G^{\prime} \rightarrow \Theta \phi_{I}$ process at $\sqrt{s}=13 \mathrm{TeV}$ as a function of the coloron mass for $\tan \theta=0.2$ (solid lines) or 0.5 (dashed lines). The scalar masses are fixed at $M_{\Theta}=1 \mathrm{TeV}$ and $M_{\phi_{I}}=300 \mathrm{GeV}$ (blue lines), or at $M_{\Theta}=M_{\phi_{I}}=2 \mathrm{TeV}$ (red lines).

\section{Production of scalars at the LHC}

The ReCoM is a relatively simple gauge theory. It contains only four new particles beyond the SM: the spin-1 coloron, the color-octet scalar $\Theta$, and two singlet scalars. Nevertheless, there are several processes at the LHC that lead to the production of these particles.

A peculiar production process is $q \bar{q} \rightarrow G^{\prime} \rightarrow \Theta \phi_{I}$, which is resonant production of two scalar particles of different masses and carrying different color charges. In the narrow width approximation, the tree-level $G^{\prime}$ parton-level production cross section is given by

$$
\sigma\left(q \bar{q} \rightarrow G^{\prime}\right) \approx \frac{8 \pi^{2} \alpha_{s} \tan ^{2} \theta}{9 M_{G^{\prime}}} \delta\left(\sqrt{\hat{s}}-M_{G^{\prime}}\right) .
$$

Next-to-leading order corrections to coloron production have been computed in [18], and can be approximated by a multiplicative $K$-factor of about 1.2 . At the $13 \mathrm{TeV}$ LHC, the $G^{\prime}$ production cross section is approximately $(20,1.8,0.20) \mathrm{pb}$ for $M_{G^{\prime}}=2,3,4 \mathrm{TeV}$. Using the MSTW parton distribution functions [19], $K=1.2$, and the branching fraction for $G^{\prime} \rightarrow \Theta \phi_{I}$ (see figure 2), we find the cross section for $\Theta \phi_{I}$ production shown in figure 5 .

The color-octet scalar $\Theta$ can be pair-produced at hadron colliders through two very different processes. The first one, which we loosely refer to as QCD production, is due to the $\Theta$ coupling to the gluons, and is governed by the QCD gauge coupling $g_{s}=\sqrt{4 \pi \alpha_{s}} \approx 1$ (here $\alpha_{s}$ is the strong coupling constant at a scale of order $2 M_{\Theta}$ ). There are four types of Feynman diagrams that contribute to QCD production at leading order [2]: three diagrams from the $g g$ initial state (via an $s$-channel gluon, a $t$-channel $\Theta$, or a $g g \Theta \Theta$ coupling) and one diagram from $q \bar{q}$ initial states (via an $s$-channel gluon). At the LHC, the gluon-initiated processes have a larger rate than the $q \bar{q}$ ones, unless $\Theta$ is very heavy.

The second process that leads to $\Theta$ pair production involves an $s$-channel coloron. At leading order this is due to quark-antiquark initial states, so it interferes only with the $q \bar{q}$ initiated QCD production (this represents between $10 \%$ and $30 \%$ of the nonresonant $\Theta \Theta$ 


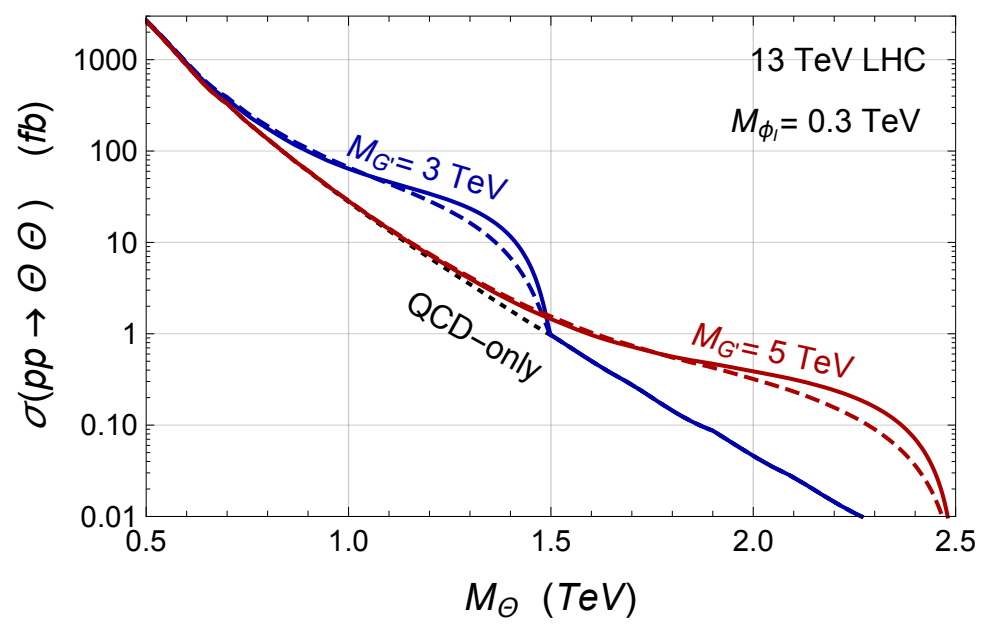

Figure 6. Leading-order cross section for $\Theta \Theta$ production (due to an $s$-channel $G^{\prime}$, QCD and interference) at the $13 \mathrm{TeV}$ LHC as a function of the $\Theta$ mass. The coloron mass is fixed at $M_{G^{\prime}}=$ $3 \mathrm{TeV}$ (blue lines) or $5 \mathrm{TeV}$ (red lines), while $\tan \theta=0.2$ (solid lines) or 0.5 (dashed lines). The dependence on the neutral-scalar mass (fixed here at $M_{\phi_{I}}=300 \mathrm{GeV}$ ) is mild. The dotted line represents the QCD contribution.

production rate for $M_{\Theta}$ in the $1-2.5 \mathrm{TeV}$ range). The interference effects are thus small, are further suppressed when the coloron is a narrow resonance, and may be relevant only for a small range of parameters where the amplitudes for QCD and resonant productions are comparable. The total $\Theta \Theta$ production cross section at the $13 \mathrm{TeV}$ LHC is shown in figure 6 as a function of the $\Theta$ mass, for $M_{G^{\prime}}=3 \mathrm{TeV}$ or $5 \mathrm{TeV}, \tan \theta=0.2$ or 0.5 , and $M_{\phi_{I}}=300 \mathrm{GeV}$. The dependence on $M_{\phi_{I}}$ (due to the $G^{\prime} \rightarrow \Theta \phi_{I}$ branching fraction) is negligible when this parameter is much smaller than $M_{\Theta}$.

There are also two classic processes, $q \bar{q} \rightarrow G^{\prime} \rightarrow j j$ and $q \bar{q} \rightarrow G^{\prime} \rightarrow t \bar{t}$, which have been searched for by the ATLAS and CMS experiments, and can be used to exclude a certain region of the parameter space. After taking into account the acceptance of around 0.6 [20], the CMS collaboration has set an upper constraint on the cross section times branching fraction $(\sigma B)$ from dijet resonance searches with $36 \mathrm{fb}^{-1}$ of data (similar constraints are also imposed by the ATLAS collaboration [21]). For $M_{G^{\prime}}=(2,3,4) \mathrm{TeV}$, the constraint is $\sigma\left(p p \rightarrow G^{\prime}\right) B\left(G^{\prime} \rightarrow j j\right) \leq(100,20,10)$ fb [20]. For a small mixing of $\tan \theta=0.2$, $B\left(G^{\prime} \rightarrow j j\right)$ could be as small as $5 \%$ such that the constraint on the coloron mass is around $M_{G^{\prime}} \gtrsim 2 \mathrm{TeV}$.

In figure 7, we show the constraints on the mixing parameter $\tan \theta$ for different coloron masses from the dijet resonance searches. We also note that the $t \bar{t}$ resonance searches are less sensitive because of the smaller branching fraction [22]. It is clear from the left panel of figure 2 that the main decay channels of the coloron for small $\tan \theta$ are into the scalars in the ReCoM, which provides novel signatures at the LHC and will be the focus of next section.

The second singlet scalar, $\phi_{R}$, cannot be produced in coloron decays, as it only couples to $G_{\mu}^{\prime} G^{\prime \mu}$. This coupling allows the non-resonant production of $\phi_{R}$ in association with a coloron. Single production of $\phi_{R}$ also proceeds through a gluon fusion process induced at 


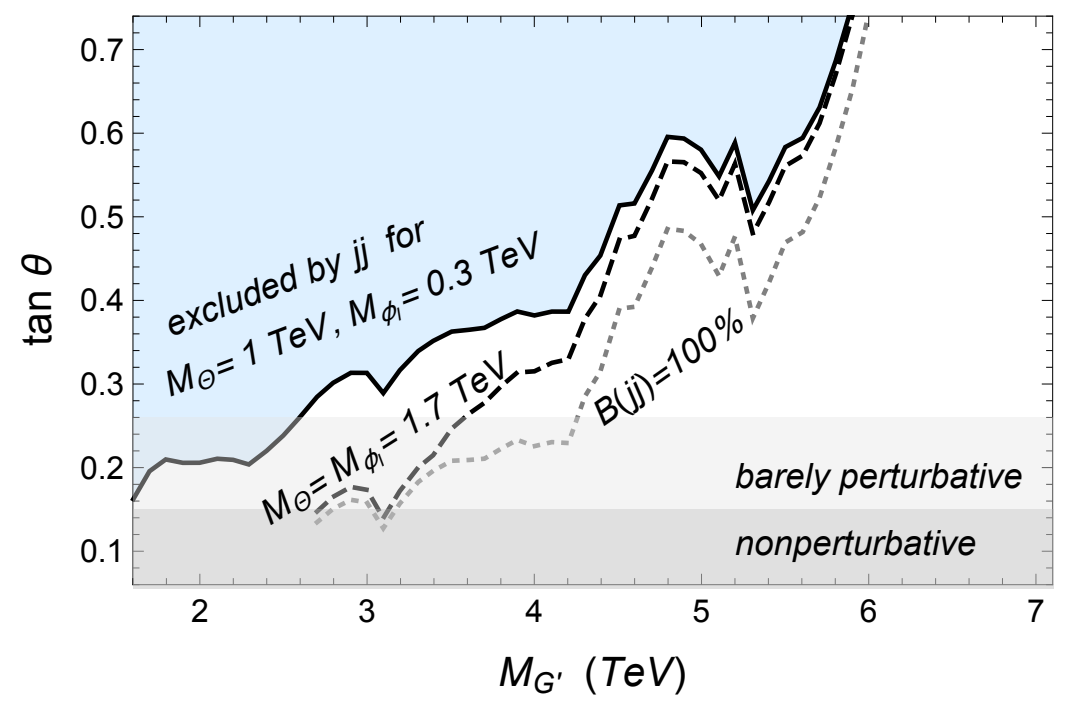

Figure 7. Excluded regions of the coloron parameter space. Region above solid black line is ruled out, for $M_{\Theta}=1 \mathrm{TeV}$ and $M_{\phi_{I}}=300 \mathrm{GeV}$, by $j j$ resonance searches, assuming that $p p \rightarrow G^{\prime} \rightarrow j j$ is the only new-physics process contributing to the dijet final state. Similarly, the region above the dashed line is ruled out for $M_{\Theta}=M_{\phi_{I}}=1.7 \mathrm{TeV}$. The region above the dotted line is ruled out when the effective $j j$ branching fraction is $100 \%$ (e.g., scalar masses are above $M_{G^{\prime}} / 2$ and the boosted $t \bar{t}$ signal mimics a $j j$ one). In the region labelled "barely perturbative", the expansion parameter for loops that involve the coloron self-coupling is relatively large $\left[3 h_{2}^{2} /\left(16 \pi^{2}\right)\right.$ between $1 / 3$ and 1] so that the tree-level result is expected to get sizable corrections.

one loop, with separate contributions from the coloron and the $\Theta$ scalar running in the loop. If $\phi_{R}$ is heavy enough, it can decay into a pair of $\phi_{I}$ or $\Theta$ scalars due to the trilinear couplings in eq. (2.12). The $\phi_{R}$ scalar may also decay into pairs of heavy SM particles due to its mixing with the SM Higgs boson, as studied in [12, 13].

Another class of processes is based on coloron pair production through its gluon couplings. Even though this production is non-resonant and kinematically suppressed by the presence of two heavy colorons, its cross section relies solely on the QCD coupling and is above $1 \mathrm{fb}$ for a coloron mass below about $2.5 \mathrm{TeV}$ [23]. Depending on the decay channels, this may be large enough for the high-luminosity run of the LHC. The coloron decays into scalar pairs in this case leads to intermediate states involving $4 \Theta$ or $3 \Theta+\phi_{I}$ or $\Theta \phi_{I} \Theta \phi_{I}$, which give rise to complicated final states with high jet multiplicity.

In the next section we will focus on the final states that arise from $\Theta \phi_{I}$ or $\Theta \Theta$ production, which have larger cross sections.

\section{Novel LHC signatures}

The ReCoM predicts rich collider phenomena with many complicated final states involving hadronic activity and other objects. At the LHC, the single production of $\Theta$ or $\phi_{I}$ is suppressed due to loop factors. Therefore, we will concentrate on the pair-productions $p p \rightarrow \Theta \Theta$ and $p p \rightarrow \Theta \phi_{I}$ and point out the novel signatures in the ReCoM. 

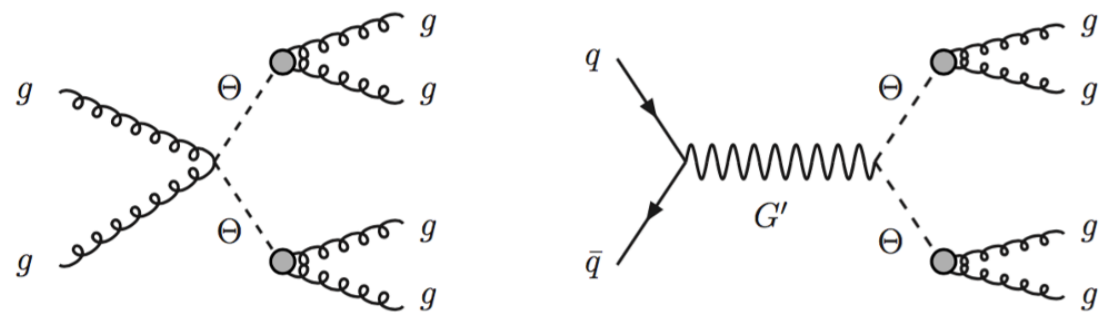

Figure 8. Pair production of the color-octet scalar $\Theta$, through QCD (left-hand diagram; related diagrams with only trilinear vertices are not shown), and coloron decay (right-hand diagram), followed by $\Theta$ decays into two gluons at one loop. The branching fraction for $\Theta \rightarrow g g$ is close to $100 \%$ when $M_{\Theta}<M_{\phi_{I}}$.

To study the current experimental bounds and interesting signatures that have not been searched for at the LHC, we consider two types of mass orderings: $M_{G^{\prime}}>M_{\phi_{I}}>M_{\Theta}$ and $M_{G^{\prime}}>M_{\Theta}>M_{\phi_{I}}$. In the following, we will study the leading collider signatures for each case and point out the discovery potential.

\subsection{Light color-octet scalar: $M_{G^{\prime}}>M_{\phi_{I}}>M_{\Theta}$}

For this parameter region, the color-octet scalar $\Theta$ mainly decays into two gluons $(\Theta \rightarrow g g)$, while the singlet scalar predominantly decays via an off-shell coloron into a 3-body final state: $\phi_{I} \rightarrow j j \Theta$ with the two jets originating from quarks. After the subsequent $\Theta$ decaying to two gluons, $\phi_{I}$ behaves as a 4 -jet resonance with a 2-body sub-resonance.

Starting from the pair production of $p p \rightarrow \Theta \Theta \rightarrow 4 g$, the final state contains four jets with a pair of dijet resonances of equal mass, as can be seen in figure 8 . This final state with a pair of dijet resonances has been studied in refs. [2, 23-25] and searched for at the LHC by both CMS [26, 27] and ATLAS [28, 29]. Using the latest result from ATLAS with $36.7 \mathrm{fb}^{-1}$ data [29], the upper limit on the cross section for production of a coloroctet scalar pair is around $0.4 \mathrm{pb}$ for $M_{\Theta}=700 \mathrm{GeV}$, which approximately matches the production cross section predicted in the ReCoM, shown in figure 6 . So, for this region of parameter space, the limit on the color-octet scalar mass is $M_{\Theta} \gtrsim 700 \mathrm{GeV}$.

As shown in figure 6 , the $\Theta$ pair production is typically dominated by the coloronmediated process when $M_{G^{\prime}}>2 M_{\Theta}$. Thus, besides searching for a pair of dijet resonances, one could also search for a resonance in the invariant mass spectrum of all four jets (for a related collider study with $b$ jets at the Tevatron, see [2]).

For a hierarchical mass spectrum with $M_{G^{\prime}} \gg M_{\Theta}$, the $\Theta$ particle from $G^{\prime}$ decays could be highly boosted and behave as a jet with 2-prong substructure at the LHC. The latter has an invariant mass given by $M_{\Theta}$, and we label it by $J_{\Theta}$. As a rough estimate, the angular separation of the two gluon-jets from $\Theta$ decays has a lower bound approximately given by [30]

$$
\Delta R_{g g} \simeq \frac{2 M_{\Theta}}{p_{T}(\Theta)} \simeq 4 \frac{M_{\Theta}}{M_{G^{\prime}}} .
$$




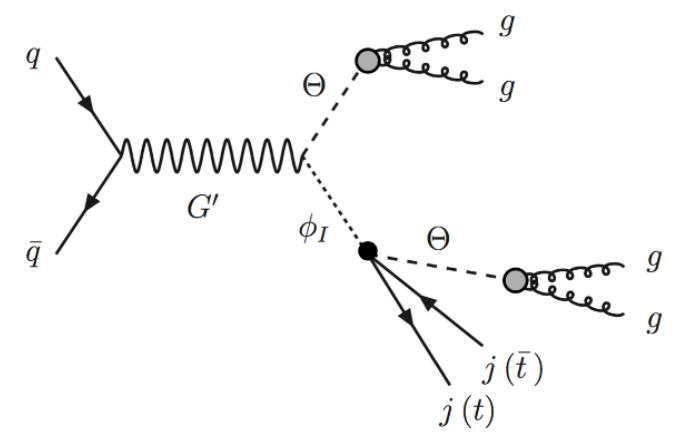

Figure 9. Resonant production of a coloron that decays into an octet scalar $\Theta$ and a singlet scalar $\phi_{I}$, which then decays (for $M_{\Theta}<M_{\phi_{I}}$ ) via an off-shell coloron into a $\Theta$ plus either two quark jets or $t \bar{t}$.

For instance, one could enlarge the jet-finding-algorithm radius to $R \sim 0.8$ for $M_{\Theta}=1 \mathrm{TeV}$ and $M_{G^{\prime}}=5 \mathrm{TeV}$ and use additional jet-substructure techniques to search for the signal in this case [31].

If the scalars are even more boosted such that the two gluon-jets are within the jet cone used in regular dijet searches, then the $p p \rightarrow G^{\prime} \rightarrow \Theta \Theta \rightarrow J_{\Theta} J_{\Theta}$ process will effectively appear as a dijet resonance. The effective dijet branching fraction of the coloron would then be larger, and the constraint from dijet resonance searches would be stronger (see figure 7). However, using a typical $\Delta R_{g g}=0.4$ in eq. (4.1) would require $M_{\Theta} / M_{G^{\prime}} \lesssim O(0.1)$. This in conjunction with the lower limit on $M_{\Theta}$ would push $M_{G^{\prime}}$ above $7 \mathrm{TeV}$, where the production cross section becomes too small.

For the singlet scalar, $\phi_{I}$, the main production is together with a $\Theta$ scalar via $G^{\prime}$ decays. The corresponding Feynman diagram for its production and decay is shown in figure 9 . When it is produced at the LHC, the $\phi_{I}$ undergoes a 3-body decay into $\Theta$ plus a quark pair via an off-shell coloron. When the quark is not the top, the quark pair hadronizes into two jets. After the two $\Theta$ 's decay, there are altogether six jets in the final state, with two of them having an invariant mass of $M_{\Theta}$ and four of them an invariant mass of $M_{\phi_{I}}: p p \rightarrow G^{\prime} \rightarrow \Theta \phi_{I} \rightarrow \Theta(\Theta q \bar{q}) \rightarrow(g g)((g g) q \bar{q})$. The total invariant mass of all six jets should match the coloron mass $M_{G^{\prime}}$. To our knowledge, there are no dedicated experimental searches to cover this signature. A less sensitive but related search is looking for microscopic black holes at the $13 \mathrm{TeV}$ LHC via multiple jets [32] with $2.3 \mathrm{fb}^{-1}$ luminosity. Based on a parton-level simulation, we conclude that the upper bound on the cross section for six or more jets does not constrain the ReCoM.

Searches for the signal of multiple resonances in 6-jet final states would suffer from a combinatorial background. For instance, there are $C_{6}^{2} C_{4}^{2}=90$ dijet pairs if the search is designed to find the two dijet resonances associated with $\Theta$. This combinatorial issue would likely make the reconstructed $\Theta$ resonance very broad.

Instead of reconstructing the $\Theta$ or $\phi_{I}$ resonances, a simple strategy is to use the invariant mass of all six jets to search for the $G^{\prime}$ resonance. Even then, additional jets from initial state radiation would complicate the reconstruction of the resonance. In addition, the jet energy resolution in the presence of a large number of jets would make the resonance broad. 
The situation is dramatically different when $M_{G^{\prime}} \gg M_{\phi_{I}}>M_{\Theta}$. The signal arising from the asymmetric decay of a coloron produced in the $s$-channel, $p p \rightarrow G^{\prime} \rightarrow \Theta \phi_{I}$, is then a "dijet" resonance, with one of the jets $\left(J_{\Theta}\right)$ having a 2-prong substructure, and the other jet (of mass $M_{\phi_{I}}$ and labelled by $J_{j j \Theta}$ ) having a 4-prong substructure. In this case, the effective dijet branching fraction is closer to $100 \%$ (provided that each multi-prong jet fits inside an $\Delta R=0.4$ cone), so that the constraint from dijet resonance searches approaches the dotted line in figure 7 .

The case where $M_{G^{\prime}}>M_{\phi_{I}} \gg M_{\Theta}$ would still be phenomenologically different. Note, however, that this case is not consistent with the upper limit $M_{\phi_{I}}<2.1 M_{\Theta}$ derived in appendix A, which is based on the assumption that the color-preserving vacuum is the global minimum of the scalar potential.

Nevertheless, there are interesting intermediate cases, where $M_{G^{\prime}}$ is considerably larger than $M_{\phi_{I}}+M_{\Theta}$ such that the primary $\Theta$ is moderately boosted, while the two quark jets and the $\Theta$ arising from the $\phi_{I}$ decay have only a small boost. An example of mass spectrum that leads to the above situation is $M_{\Theta} \approx 1 \mathrm{TeV}, M_{\phi_{I}} \approx 2 \mathrm{TeV}$ and $M_{G^{\prime}} \approx 6 \mathrm{TeV}$. The signal is a $J_{\Theta}$, which in this case is a wide jet of $\Delta R_{g g} \approx 0.8$, and four other jets that reconstruct the $\phi_{I}$ mass. The reconstruction of the resonances would be improved by techniques that distinguish between gluon jets and quark jets.

A separate class of signatures arises from the $\phi_{I} \rightarrow t \bar{t} \Theta$ decay (see figure 9). Even though the branching fraction for this 3-body decay is $1 / 6$, or smaller when $M_{\phi_{I}}-M_{\Theta}$ is near $2 m_{t}$, the more complicated final states associated with top quarks may be used to reduce the backgrounds. For example, one may use the leptonic decay of a $W$ from the $p p \rightarrow G^{\prime} \rightarrow \phi_{I} \Theta \rightarrow W^{+} W^{-} b \bar{b}+4 j$ process.

If $M_{G^{\prime}} \gg M_{\phi_{I}}>M_{\Theta}$, then the $\phi_{I} \rightarrow t \bar{t} \Theta \rightarrow t \bar{t} j j$ system is also boosted, giving rise to an object (we label it by $J_{t \bar{t} \Theta}$ ) that includes multi-prong jet substructure and sometimes nonisolated leptons and missing energy. The signal is then $p p \rightarrow G^{\prime} \rightarrow J_{\Theta} J_{t \bar{t} \Theta}$.

\subsection{Light singlet scalar: $M_{G^{\prime}}>M_{\Theta}>M_{\phi_{I}}$}

Let us now study the mass ordering where the singlet scalar $\phi_{I}$ is lighter than the coloroctet scalar $\Theta$. $\Theta$ has 2-body decays into two gluons and 3-body decays into $j j \phi_{I}$ with the branching fractions depending on $\tan \theta$ and on the $M_{\Theta} / M_{G^{\prime}}$ and $M_{\phi_{I}} / M_{G^{\prime}}$ mass ratios, as follows from eqs. (2.20) and (2.21) (see also figure 1).

The $\phi_{I}$ undergoes 4-body 1-loop decays into two gluons and a quark-antiquark pair, or 2-body 3-loop decays into $W^{+} W^{-}, \gamma Z$ or $Z Z$. The latter are dominant for $M_{\phi_{I}}<M_{0}$ where the mass scale $M_{0}$ can be derived from eq. (2.37):

$$
M_{0} \approx 0.7 \eta_{3}^{1 / 4}\left(\tan \theta M_{\Theta} M_{G^{\prime}}\right)^{1 / 2}
$$

As mentioned in section 2.4, $\eta_{3}$ is a number that accounts for the 3-loop integrals, which are difficult to calculate; we expect $\eta_{3}$ to be of order 1 , but values as low as $1 / 30$ or so would not be very surprising. 

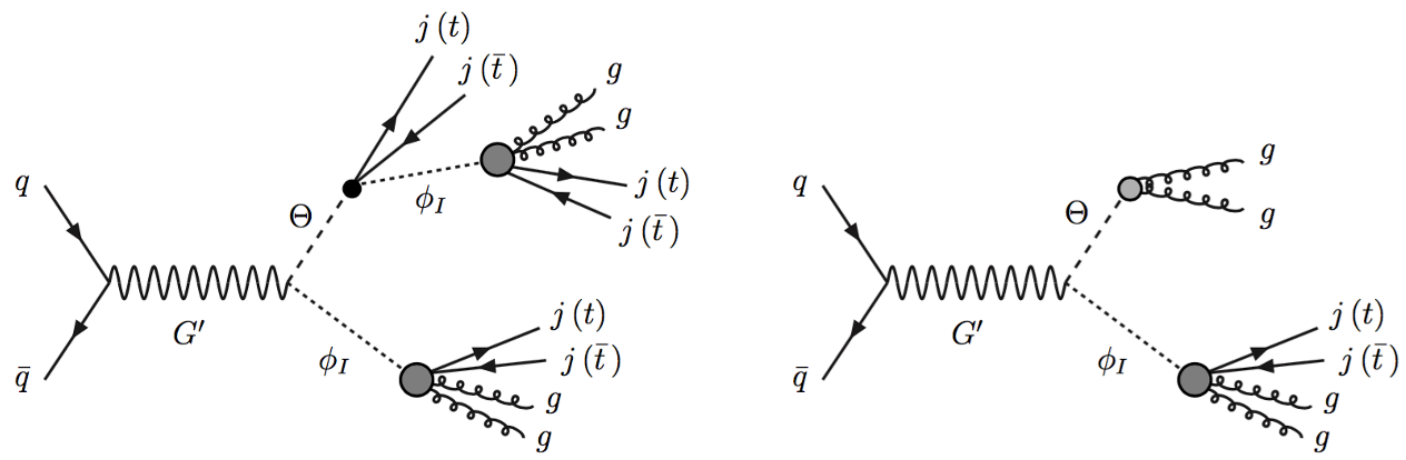

Figure 10. Asymmetric $p p \rightarrow G^{\prime} \rightarrow \phi_{I} \Theta$ processes for $M_{\Theta}>M_{\phi_{I}}$, when the $\phi_{I}$ scalar decays into two gluons plus two quarks, which occurs at one-loop (see figure 3). Left diagram: scalar pair production is followed by a 3-body decay of the color-octet $\Theta$ into $\phi_{I}$ plus a quark-antiquark pair, which forms two jets, or less often a $t \bar{t}$ pair. Right diagram: $\Theta$ decays into two gluons, at one loop.

\subsection{1 $\quad \phi_{I} \rightarrow g g q \bar{q}$}

Let us first focus on the $M_{\phi_{I}}>M_{0}$ case, implying that $\phi_{I}$ decays predominantly into $g g q \bar{q}$. An interesting process (see right diagram of figure 10) is $s$-channel coloron production followed by the asymmetric coloron decay into $\Theta \phi_{I}$, and leading to a ten jet final state: $p p \rightarrow G^{\prime} \rightarrow \Theta \phi_{I} \rightarrow\left(\phi_{I} q \bar{q}\right) \phi_{I} \rightarrow((g g q \bar{q}) q \bar{q})(g g q \bar{q}) \rightarrow 10 j$. Each parenthesis here denotes the presence of a resonance.

Depending on the masses of the three ReCoM bosons, some of these jets may be merged. In particular, when $M_{G^{\prime}}$ and $M_{\Theta}$ have the same order of magnitude while the scalar masses satisfy $M_{\Theta} \gg M_{\phi_{I}}$ and $M_{G^{\prime}}-M_{\Theta} \gg M_{\phi_{I}}$, the final state includes four jets with two of them having each a 4-prong substructure and an invariant mass given by $M_{\phi_{I}}$. We use the notation $J_{\phi_{I}}$ for such a jet formed by the merger of two gluons and two quark jets arising from a $\phi_{I}$ decay. The above cascade decay of the coloron is then $G^{\prime} \rightarrow \Theta \phi_{I} \rightarrow J_{\phi_{I}} J_{\phi_{I}} j j$. If $M_{G^{\prime}}-M_{\Theta}$ is near $M_{\phi_{I}}$ (a more tuned situation) and $M_{\Theta} \gg M_{\phi_{I}}$, then the signal consists of seven jets, with only one of them being an $J_{\phi_{I}}$. For $M_{G^{\prime}} \gg M_{\Theta}>M_{\phi_{I}}$, the whole $q \bar{q} \phi_{I} \rightarrow q \bar{q}\left(g g q^{\prime} \bar{q}^{\prime}\right)$ system (labelled by $J_{j j \phi_{I}}$ ) is boosted, and is approximately back-to-back against a $J_{\phi_{I}}$ (we will see though below that the branching fraction for $\Theta \rightarrow q \bar{q} \phi_{I}$ is small in this case).

There is also a $p p \rightarrow G^{\prime} \rightarrow \Theta \phi_{I} \rightarrow(g g)(g g q \bar{q}) \rightarrow 6 j$ process, where two gluon jets reconstruct the $\Theta$ mass, and the other four jets reconstruct the $\phi_{I}$ mass. So far, no search in the six jet final state has been performed by imposing a separate mass constraint for two different resonances. Even though the intermediate states are different than in the process shown in figure 9 and discussed in section 4.1, the final state is identical. Nevertheless, the range of parameters here is larger than in the case discussed in section 4.1, leading to novel boosted topologies. For $M_{G^{\prime}}>M_{\Theta} \gg M_{\phi_{I}}$ the resonant signal has three jets, with one of them being an $J_{\phi_{I}}$, and the other two jets forming a resonance at $M_{\Theta}$. Requiring the total invariant mass of three jets match the $G^{\prime}$ mass should provide a sensitive way to test these signatures. Using a simple estimate where the system of the four partons is boosted in the 
shape of a tetrahedron, we find that $\phi_{I} \rightarrow g g q \bar{q}$ fits inside a cone of

$$
\Delta R_{g g q \bar{q}} \simeq \frac{2 \sqrt{2} M_{\phi_{I}}}{p_{T}\left(\phi_{I}\right)} \simeq \frac{4 \sqrt{2} M_{\phi_{I}}}{M_{G^{\prime}}\left(1-M_{\Theta}^{2} / M_{G^{\prime}}^{2}\right)} .
$$

Thus, one could identify the $J_{\phi_{I}}$ jet using a normal jet-finding radius $R \sim 0.8$ for $M_{\phi_{I}} \approx$ $0.8 \mathrm{TeV}, M_{\Theta} \approx 1 \mathrm{TeV}$ and $M_{G^{\prime}} \approx 6 \mathrm{TeV}$.

The quark-antiquark pair arising from either $\Theta \rightarrow q \bar{q} \phi_{I}$ or $\phi_{I} \rightarrow g g q \bar{q}$ decays may be a $t \bar{t}$ pair, albeit the branching fraction is only $1 / 6$ (or smaller if there is kinematic suppression), as opposed to $5 / 6$ for light SM quarks. In the case of the $G^{\prime} \rightarrow \Theta \phi_{I} \rightarrow$ $\left(\phi_{I} q \bar{q}\right) \phi_{I} \rightarrow((g g q \bar{q}) q \bar{q})(g g q \bar{q})$ cascade decay, the probability of having one of the quarkantiquark pairs to be $t \bar{t}$ can be as large as $34.7 \%$, and even the case where there are two $t \bar{t}$ pairs is potentially relevant, having a probability of $6.9 \%$ (without taking into account the $G^{\prime}$ and $\Theta$ branching fractions). Thus, if there are no large mass hierarchies, the signatures include $t \bar{t}+8 j$ and $4 t+6 j$. If $M_{\phi_{I}} \ll M_{G^{\prime}}$, then the final states include $J_{\phi_{I}}$ jets with 4-prong substructure, or boosted $t \bar{t} g g$ systems (we label them by $J_{t \bar{t} g g}$ ) with an invariant mass equal again to $M_{\phi_{I}}$. The latter have various interesting signatures; for example, when only one of the $W$ bosons produced by the top quarks decays leptonically, the boosted $t \bar{t} g g \rightarrow \ell b \bar{b}+4 j+E_{T}$ system will include a non-isolated lepton aligned with the missing energy. Even more complicated objects occur when $M_{G^{\prime}} \gg M_{\Theta}>M_{\phi_{I}}$. A boosted $\Theta \rightarrow t \bar{t}+4 j$ (or less often $\Theta \rightarrow 4 t+j j$ ) system is then produced approximately back-to-back with a $J_{t \bar{t} g g}$.

The main LHC signatures arising from the asymmetric $p p \rightarrow G^{\prime} \rightarrow \Theta \phi_{I}$ process are summarized in figure 11 (also including channels discussed in section 4.1). Note that some final states that require parameter tuning, or have smaller branching fractions, are not shown there.

For $2 M_{\Theta}>M_{G^{\prime}}>M_{\Theta}+M_{\phi_{I}}$, the asymmetric channel $G^{\prime} \rightarrow \Theta \phi_{I}$ is open and has a large branching fraction, while the symmetric $G^{\prime} \rightarrow \Theta \Theta$ channel is kinematically closed (even then, the QCD production of a pair of $\Theta$ scalars leads to many of the additional final states discussed in what follows).

For $M_{G^{\prime}}>2 M_{\Theta}>M_{\Theta}+M_{\phi_{I}}$, both the asymmetric and the symmetric channels are open. Note that there is interference between the $p p \rightarrow G^{\prime} \rightarrow \Theta \Theta$ and QCD $\Theta$ pair production. The symmetric coloron decays lead to a variety of signatures in addition to those from figure 11. Pair production of the color-octet scalar followed by each $\Theta$ decaying into $j j \phi_{I}$ leads to a twelve jet final state: $p p \rightarrow \Theta \Theta \rightarrow \phi_{I} \phi_{I}+4 j \rightarrow 12 j$ (see the left diagram of figure 12) if the main decay of $\phi_{I}$ is $\phi_{I} \rightarrow g g q \bar{q}$ at one loop. The searches for microscopic black holes via multi-jet final states [32] may become sensitive to this ReCoM signal after the LHC accumulates enough luminosity. Specific to ReCoM, searching for a resonance in the invariant mass spectrum of all twelve jets can substantially improve the search sensitivity.

For the parameter region with $M_{\Theta} \gg M_{\phi_{I}}$, the $\phi_{I}$ from $\Theta$ decays is likely to be boosted. Thus, the signature is $p p \rightarrow \Theta \Theta \rightarrow J_{\phi_{I}} J_{\phi_{I}}+4 j$. A possible search strategy is to require two jets to have a 4-prong substructure and approximately the same mass. In 


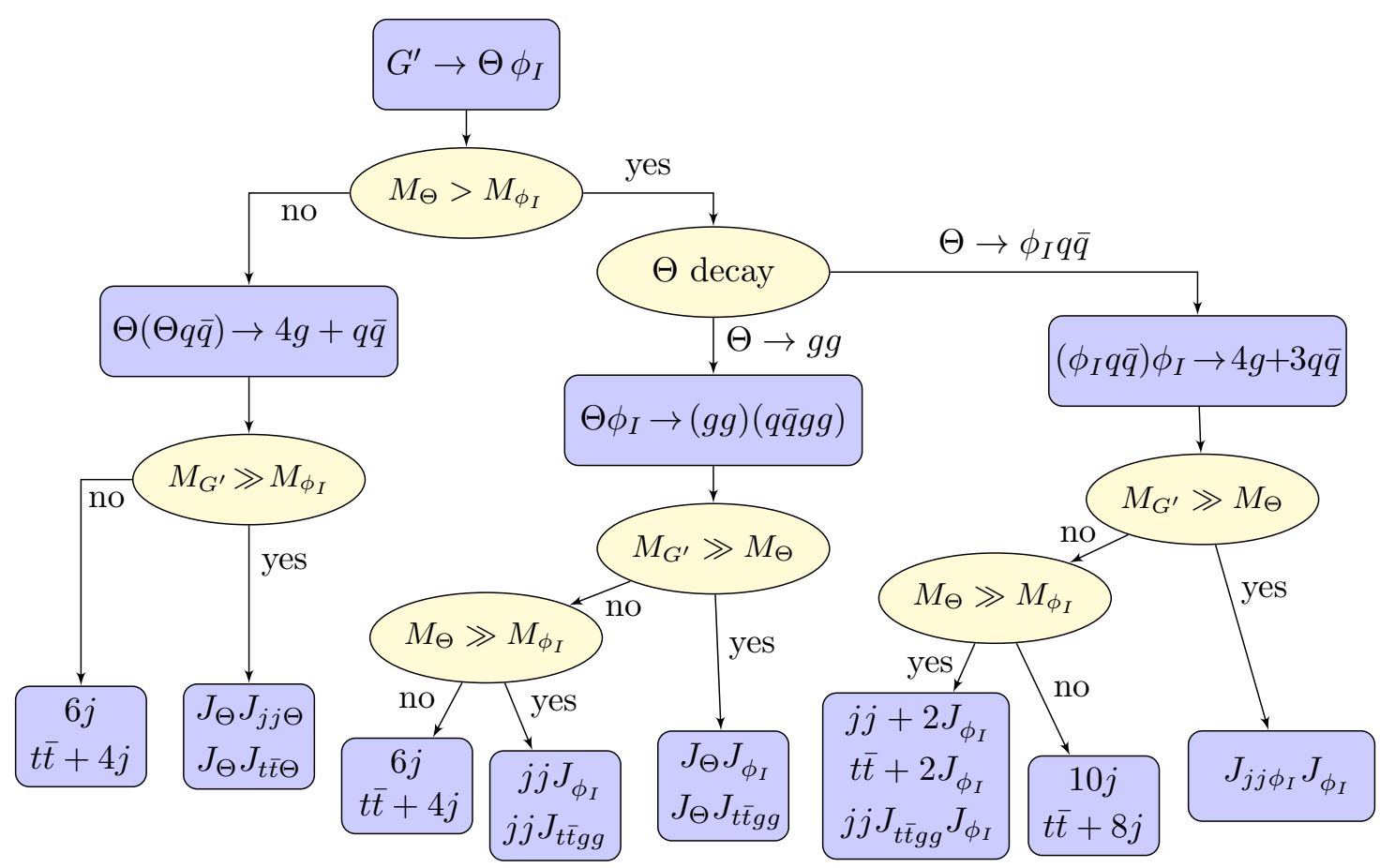

Figure 11. Signatures of the ReCoM from the asymmetric $p p \rightarrow G^{\prime} \rightarrow \Theta \phi_{I}$ production, and the corresponding mass relations. For $M_{\phi_{I}}<M_{\Theta}$, only the 1-loop $\phi_{I} \rightarrow g g q \bar{q}$ decays are included here. The jet labelled by $J_{\Theta}$ has a 2-prong substructure, while $J_{j j \phi_{I}}$ has a 6 -prong substructure; both have an invariant mass given by $M_{\Theta}$. The jets labelled by $J_{\phi_{I}}$ and $J_{j j \Theta}$ have a 4-prong substructure and an $M_{\phi_{I}}$ invariant mass. The boosted objects labelled by $J_{t \bar{t} g g}$ and $J_{t \bar{t} \Theta}$ have more complicated substructure and an $M_{\phi_{I}}$ invariant mass. For $M_{\phi_{I}} \lesssim O(1 \mathrm{TeV})$ and $M_{\phi_{I}}<M_{\Theta}, \phi_{I}$ is so long-lived that $J_{\phi_{I}}$ and $J_{t \bar{t} g g}$ may have a displaced origin.

addition, one may try to reconstruct the $\Theta$ mass from the $C_{4}^{2}=6$ pairs of 3-jet resonances, similar to the searches for gluinos undergoing $R$ parity violating decays [33-35].

If the $\Theta \rightarrow \phi_{I} q \bar{q}$ and $\Theta \rightarrow g g$ decays have comparable branching fractions, then the process $p p \rightarrow \Theta \Theta \rightarrow\left(\phi_{I} q \bar{q}\right)(g g) \rightarrow 8 j$ (see the right diagram of figure 12) has a large rate. For $M_{\Theta} \gg M_{\phi_{I}}$, the final state becomes $J_{\phi_{I}}+4 j$. This has the same combinatorial factor of 6 for reconstructing the intermediate $\Theta$ resonance.

A substantial 3-body branching fraction for $\Theta$ requires that the mass ratio of $M_{\Theta} / M_{G^{\prime}}$ is not too small (see figure 1). As a result, the 3-body decaying $\Theta$ is less likely to be boosted. To be more precise, for $M_{\phi_{I}} \ll M_{\Theta} \ll M_{G^{\prime}}$ eqs. (2.20) and (2.21) imply a ratio of branching fractions

$$
\begin{aligned}
\frac{B\left(\Theta \rightarrow \phi_{I} j j, \phi_{I} t \bar{t}\right)}{B(\Theta \rightarrow g g)} & \simeq \frac{4 \pi \tan ^{2} \theta}{405 \alpha_{s}\left(\pi^{2} / 9-1\right)^{2}\left(1+r_{\mathcal{A}}\right)^{2}}\left(\frac{M_{\Theta}}{M_{G^{\prime}}}\right)^{2} \\
& \approx 0.1\left(\frac{\tan \theta}{0.4}\right)^{2}\left(\frac{M_{\Theta}}{1 \mathrm{TeV}}\right)^{2}\left(\frac{5 \mathrm{TeV}}{M_{G^{\prime}}}\right)^{2}
\end{aligned}
$$

where the 3-body branching fraction is summed over all six flavors and $r_{\mathcal{A}} \approx 0.53$. The three jets produced in the $\Theta \rightarrow \phi_{I} q \bar{q} \rightarrow J_{\phi_{I}} j j$ decay fit inside an $\Delta R=0.8$ cone provided 

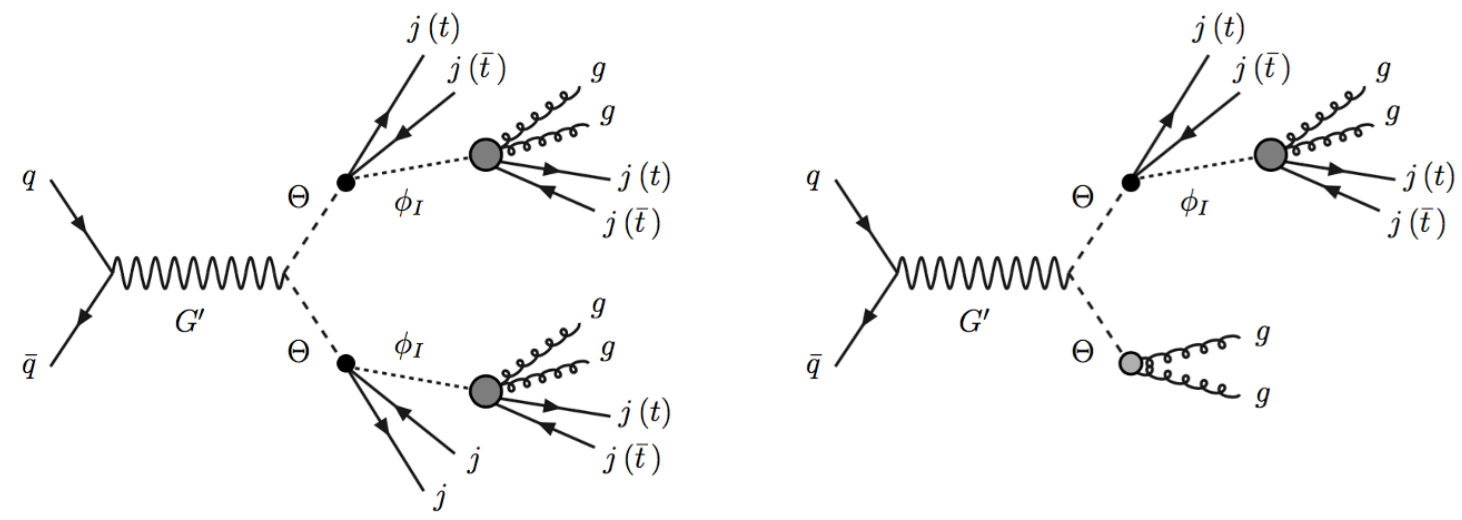

Figure 12. Resonant production of a coloron that decays into a pair of color-octet scalars, for $M_{\Theta}>M_{\phi_{I}}$ when the singlet scalar decays into two gluons plus two quarks. Left diagram: both $\Theta$ scalars undergo a cascade decay through an off-shell coloron. Right diagram: one $\Theta$ has a cascade decay, the other one decays at 1-loop into two gluons.

$0.8 \gtrsim \sqrt{3} M_{\Theta} / p_{T}(\Theta) \simeq 2 \sqrt{3} M_{\Theta} / M_{G^{\prime}}$, so that eq. (4.4) implies an upper limit on the 3-body branching fraction, $B\left(\Theta \rightarrow \phi_{I} j j, \phi_{I} t \bar{t}\right) \lesssim 12 \%$ for $\tan \theta=0.4$. Note that at this point in parameter space, which is almost optimal for a boosted $\Theta$ undergoing a 3body decay, the branching fractions of $G^{\prime}$ into scalars are only $B\left(G^{\prime} \rightarrow \Theta \Theta\right)=16 \%$ and $B\left(G^{\prime} \rightarrow \Theta \phi_{I}\right)=32 \%$.

In the processes discussed above, each of the quark-antiquark pairs from the $\Theta$ and $\phi_{I}$ decays may be a $t \bar{t}$ pair, with a branching fraction of $1 / 6$ (for $M_{\Theta}-M_{\phi_{I}} \gg 2 m_{t}$ and $M_{\phi_{I}} \gg 2 m_{t}$ ). Thus, the process where both $\Theta$ 's undergo cascade decays (left diagram in figure 12) includes a single $t \bar{t}$ pair with a probability of $38.6 \%$, and exactly two $t \bar{t}$ pairs with a probability of $11.6 \%$.

Pair production of $\Theta$ followed by both $\Theta$ 's decaying to $g g$ has been discussed in section 4.1. The main signatures of ReCoM arising from the $G^{\prime} \rightarrow \Theta \Theta$ decays are summarized in figure 13. Certain processes with smaller branching fractions, such as $\Theta \Theta \rightarrow\left(\phi_{I} t \bar{t}\right)\left(\phi_{I} t \bar{t}\right)$, are not included there.

The $\phi_{I} \rightarrow g g q \bar{q}$ decays are either prompt or displaced depending on the values of the four parameters $\left(M_{\phi_{I}}, M_{\Theta}, M_{G^{\prime}}, \tan \theta\right)$ that enter the 4-body width given in eqs. (2.28) and (2.26). The proper decay length of $\phi_{I}$ is approximately proportional to $M_{G^{\prime}}^{6} M_{\Theta}^{4} / M_{\phi_{I}}^{11}$, assuming that there is no fine tuning of the $M_{\Theta} / M_{\phi_{I}}$ ratio. A $\phi_{I}$ produced in coloron decays has a boost $\gamma_{\phi_{I}}=E_{\phi_{I}} / M_{\phi_{I}}$, where $E_{\phi_{I}}$ is the energy of a $\phi_{I}$ in the lab frame. The decay length of $\phi_{I}$ in the lab frame is then

$$
\begin{aligned}
\lambda_{4}\left(\phi_{I}\right) & \approx \frac{663(4 \pi)^{4} \tan ^{2} \theta}{\alpha_{s}^{5}\left(1+\tan ^{2} \theta\right)^{4}} \frac{M_{G^{\prime}}^{6} M_{\Theta}^{4}}{M_{\phi_{I}}^{12}} E_{\phi_{I}} \\
& =O(0.03 \mathrm{~cm})\left(\frac{\tan \theta}{0.3}\right)^{2}\left(\frac{700 \mathrm{GeV}}{M_{\phi_{I}}}\right)^{12}\left(\frac{M_{G^{\prime}}}{3 \mathrm{TeV}}\right)^{7}\left(\frac{M_{\Theta}}{1 \mathrm{TeV}}\right)^{4} \frac{E_{\phi_{I}}}{M_{G^{\prime}} / 6} .
\end{aligned}
$$

In the last line we assumed $\tan ^{2} \theta \ll 1$. Note that the coloron is expected to be produced with small momentum, so the process $p p \rightarrow G^{\prime} \rightarrow \Theta \Theta \rightarrow\left(\phi_{I} q \bar{q}\right)\left(\phi_{I} q \bar{q}\right)$ leads to a $\phi_{I}$ energy 


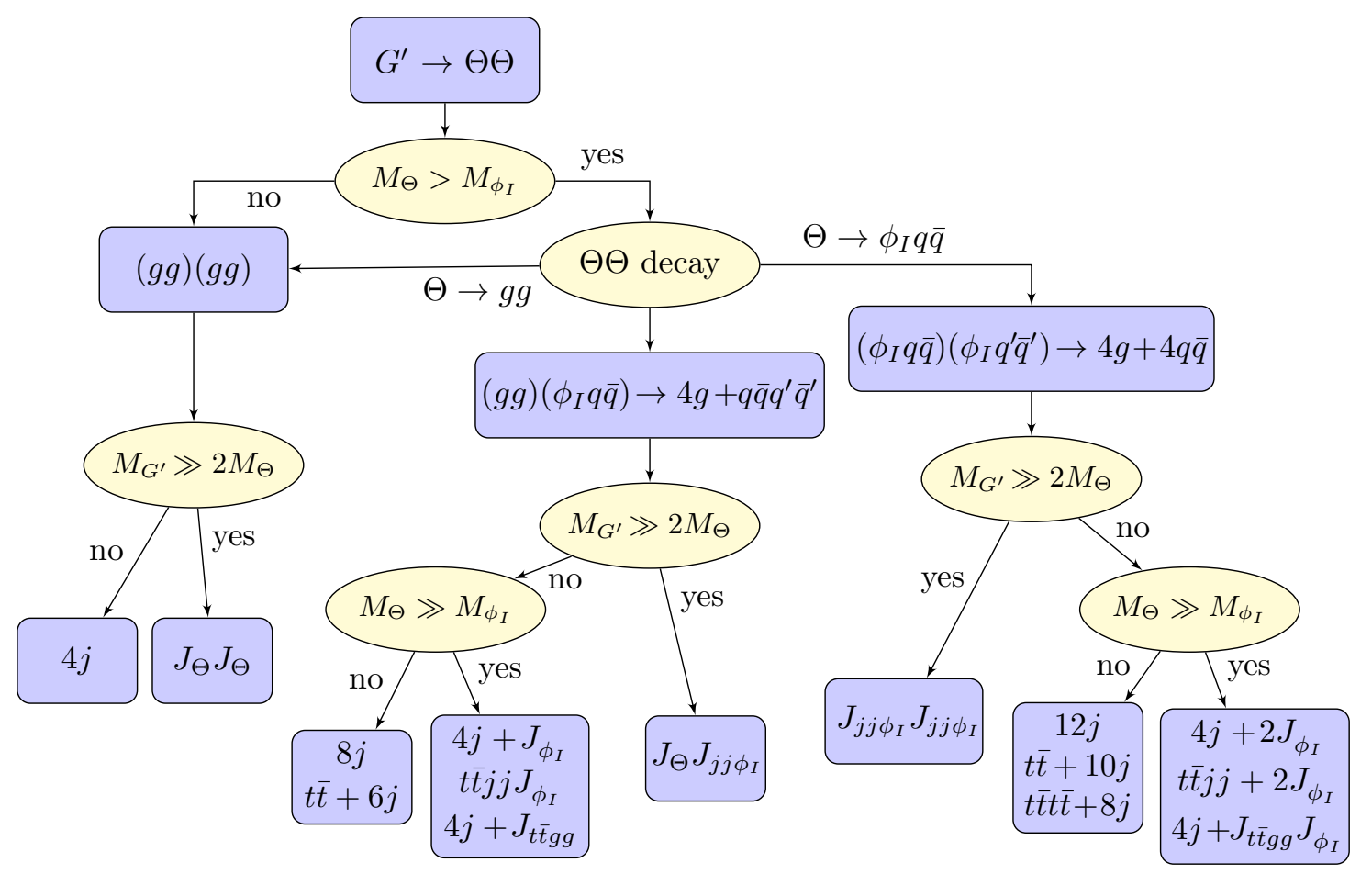

Figure 13. Same as figure 11, except that the final states shown here arise from the $p p \rightarrow G^{\prime} \rightarrow$ $\Theta \Theta$ process.

$E_{\phi_{I}} \sim O\left(M_{G^{\prime}} / 6\right)$ for a typical event, while the process $p p \rightarrow G^{\prime} \rightarrow \Theta \phi_{I} \rightarrow\left(\phi_{I} q \bar{q}\right) \phi_{I}$ leads to a slightly larger $E_{\phi_{I}}$.

The existing searches for displaced dijet resonances set an upper limit on the production cross section of a pair of displaced jets in the range of 1-10 fb for decay lengths larger than $O(1) \mathrm{mm}$ and smaller than a meter or so [36] at the $13 \mathrm{TeV}$ LHC. As this topology occurs in the ReCoM for $M_{G^{\prime}} \gtrsim 5 \mathrm{TeV}$, for example from the $G^{\prime} \rightarrow \Theta \phi_{I} \rightarrow j j J_{\phi} J_{\phi}$ process with displaced $J_{\phi}$, the production cross section shown in figure 5 is not yet constrained.

\subsection{2 $\phi_{I} \rightarrow W^{+} W^{-}, \gamma Z, Z Z$}

When $\phi_{I}$ is lighter than both $\Theta$ and the scale $M_{0}$ introduced in eq. (4.2), its leading decays are into two electroweak gauge bosons (with the exception of two photons) and occur at three loops, as in the right-hand diagram in figure 4. To find the LHC signatures, one can repeat the previous discussion with the 4-body decay $\phi_{I} \rightarrow g g q \bar{q}$ replaced by the 2-body ones. The branching fractions of $\phi_{I} \rightarrow W^{+} W^{-}, \gamma Z, Z Z$ are $65 \%, 19 \%, 16 \%$, respectively, or smaller if the $\phi_{I} \rightarrow g g q \bar{q}$ width is not negligible.

The signatures of asymmetric coloron decays are $G^{\prime} \rightarrow \Theta \phi_{I} \rightarrow\left(\phi_{I} q \bar{q} / t \bar{t}\right) \phi_{I} \rightarrow$ $2\left(\gamma Z / Z Z / W^{+} W^{-}\right)+j j / t \bar{t}$ when $\Theta$ has 3-body decays, and $\Theta \phi_{I} \rightarrow\left(\gamma Z / Z Z / W^{+} W^{-}\right)+j j$ in the case of $\Theta \rightarrow g g$. Pair production of the color-octet scalars, through QCD and $s$ channel $G^{\prime}$, leads to $\Theta \Theta \rightarrow\left(\phi_{I} q \bar{q}\right)\left(\phi_{I} q \bar{q}\right) \rightarrow 2\left(\gamma Z / Z Z / W^{+} W^{-}\right)+4 j$ and the same with one or two $q \bar{q}$ pairs replaced by $t \bar{t}$ pairs, as well as $\Theta \Theta \rightarrow\left(\phi_{I} q \bar{q}\right)(g g) \rightarrow\left(\gamma Z / Z Z / W^{+} W^{-}\right)+4 j$, 

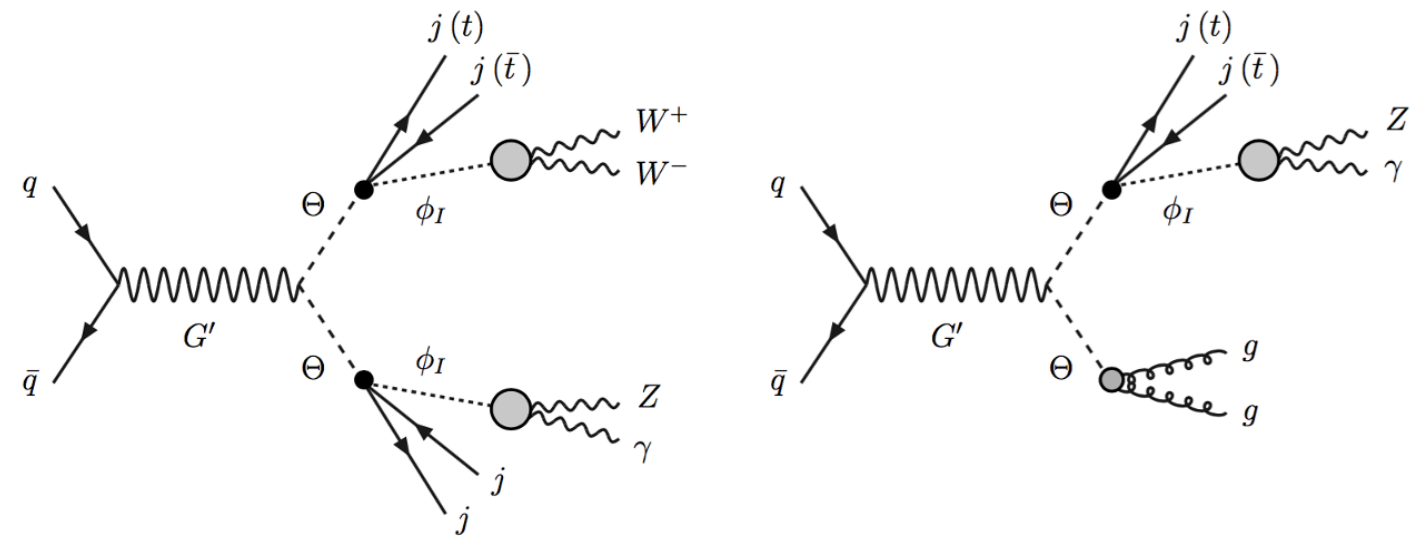

Figure 14. Representative LHC processes from symmetric coloron decays, for $M_{\Theta}>M_{\phi_{I}}$ when $\phi_{I}$ decays into pairs of electroweak bosons at three loops (represented by the largest gray disc). The $\gamma Z$ and $W^{+} W^{-}$systems are likely to be boosted and to originate from displaced vertices.

as shown for example in figure 14. Note that the boson pairs tend to be boosted given that $M_{\phi_{I}} \lesssim 1 \mathrm{TeV}$ in this case, while the coloron has a mass of several $\mathrm{TeV}$. We will use the notation $J_{W W}, J_{\gamma Z}$ and $J_{Z Z}$ for a boosted di-boson system of invariant mass equal to $M_{\phi_{I}}$, independent of how the $W$ or $Z$ bosons decay.

For hadronic decays of $Z Z$ and $W^{+} W^{-}, \phi_{I}$ could still behave as a 4-prong jet with two internal $Z$ or $W$-jets. For the final states with a photon or leptonic decays of $Z$ and $W$ bosons, the collider signatures contain $\gamma \ell^{+} \ell^{-}+$jets, $\gamma+E_{T}+$ jets, $\ell^{ \pm}+E_{T}+$ jets, $\ell^{+} \ell^{-}+E_{T}$ + jets, or more leptons when there are up to four weak bosons from two $\phi_{I}$ decays.

The 2-body partial widths of $\phi_{I}$, given in eq. (2.36), imply that the lab-frame decay length of $\phi_{I}$ in this case is

$$
\begin{aligned}
\lambda_{2}\left(\phi_{I}\right) & \approx \frac{4(4 \pi)^{6} c_{W}^{4} s_{W}^{4}}{25 \eta_{3}^{2} \alpha^{2} \alpha_{s}^{5} \tan ^{2} \theta\left(1+\tan ^{2} \theta\right)^{4}\left(3 c_{W}^{4}+s_{W}^{4}\right)} \frac{M_{G^{\prime}}^{2}}{M_{\phi_{I}}^{4}} E_{\phi_{I}} \\
& =O(0.03 \mathrm{~cm})\left(\frac{\tan \theta}{0.3}\right)^{2}\left(\frac{700 \mathrm{GeV}}{\sqrt{\eta_{3}} M_{\phi_{I}}}\right)^{4}\left(\frac{M_{G^{\prime}}}{3 \mathrm{TeV}}\right)^{3} \frac{E_{\phi_{I}}}{M_{G^{\prime}} / 6} .
\end{aligned}
$$

Note the milder dependence on $M_{\phi_{I}}$ compared to eq. (4.5), as well as the dependence on the numerical coefficient $\eta_{3}$. In most cases the $\phi_{I} \rightarrow W^{+} W^{-}, \gamma Z, Z Z$ decays are associated with displaced vertices. For $\eta_{3}$ of order one, the displacement varies between the millimeter scale (for $\phi_{I} \gtrsim 500 \mathrm{GeV}$ ) and the meter scale (for $\phi_{I} \approx 100 \mathrm{GeV}$ ), while for $\eta_{3}$ of order 0.1 the displacement grows by two orders of magnitude. Thus, we expect $W W, \gamma Z$ and $Z Z$ resonances which are both boosted and displaced. So far there have been no dedicated collider searches for boosted systems with displaced vertices (e.g., current searches for long-lived gluinos [37, 38] do not cover boosted signatures).

The $\phi_{I} \rightarrow \gamma Z$ decay may in principle be constrained by existing searches with nonpointing photons without special requirement of jet multiplicity [39]. For the lifetime of the long-lived particle in the range of $250 \mathrm{ps}$ to about $100 \mathrm{~ns}$, the upper limit on the signal production cross section at the $8 \mathrm{TeV}$ LHC ranges from $1 \mathrm{fb}$ to $100 \mathrm{fb}$. By comparing to the 
$13 \mathrm{TeV}$ LHC cross section for the production of a $\Theta$ pair shown in figure 6 , and multiplying by the branching fractions for one of the $\Theta$ 's to decay into $\phi q \bar{q}$ and also for $\phi_{I} \rightarrow \gamma Z$, we derive a lower limit on $M_{\Theta}$ of roughly $0.7 \mathrm{TeV}$ when the coloron is not too heavy [so that the 3 -body $\phi$ branching fraction is not too suppressed, see eq. (4.4)]. The signal sensitivity can be improved by also requiring the presence of high $p_{T}$ jets. Therefore, for a $\phi_{I}$ mass below about $700 \mathrm{GeV}$, searches with a displaced $J_{\gamma Z}$ and prompt high- $p_{T}$ jets could lead to a clean discovery.

When $M_{\phi_{I}}<M_{Z}$, which is a natural region of parameter space given that $\phi_{I}$ is a pseudo-Nambu Goldstone boson, the main decays of $\phi_{I}$ are 3-loop 3-body processes, which include a photon and proceed via an off-shell $Z$ boson: $\phi_{I} \rightarrow \gamma j j, \gamma \nu \bar{\nu}, \gamma \ell^{+} \ell^{-}$. The decay length is then longer than the detector, so $\phi_{I}$ behaves as a missing particle in collider experiments. The collider signatures of coloron decays then include missing transverse energy plus prompt jets or top quark pairs when there are 3-body $\Theta$ decays, or plus a dijet resonance when one of the $\Theta$ decays into two gluons.

\section{Discussion and conclusions}

The ReCoM is a UV-complete gauge extension of QCD. The sector that spontaneously breaks the $\mathrm{SU}(3)_{1} \times \mathrm{SU}(3)_{2}$ gauge symmetry includes a color-octet scalar $(\Theta)$ and two singlet scalars $\left(\phi_{I}\right.$ and $\left.\phi_{R}\right)$. It is natural that $\Theta$ and $\phi_{I}$ are lighter than the coloron, as they are pNGB's associated with an $\mathrm{SO}(18) \rightarrow \mathrm{SO}(17)$ global symmetry breaking. In addition, $\phi_{I}$ is the pNGB associated with the breaking of a global $\mathrm{U}(1)_{\Sigma}$ symmetry.

All the SM quarks are triplets under one (the first, by convention) of the $\mathrm{SU}(3)$ gauge groups, so the coloron has flavor-universal couplings. The ReCoM belongs to a small but remarkable class of gauge extensions of the SM that does not require anomalons, i.e., fermions beyond the SM quarks and leptons that cancel the gauge anomalies. ${ }^{2}$

As there are no significant constraints on the coloron from flavor-changing processes ${ }^{3}$ or electroweak observables, the only relevant limits on the coloron mass $\left(M_{G^{\prime}}\right)$ and coupling $(\tan \theta)$ are set by searches at the LHC. Dijet resonance searches set the limits in the $(\tan \theta$, $M_{G^{\prime}}$ ) plane shown in figure 7 . The $t \bar{t}$ resonance searches are weaker because the coloron branching fraction into $t \bar{t}$ is smaller than the $j j$ one by a factor of 5 .

For $\tan \theta \lesssim 0.45$, the dominant coloron decays are into a pair of scalars. The $G^{\prime} \rightarrow \Theta \phi_{I}$ and $\Theta \Theta$ decays have typically the largest branching fractions (unless the masses are near the kinematic threshold, see figure 2). The subsequent decays of $\Theta$ and $\phi_{I}$ lead to a variety of final states, depending on four parameters: $M_{G^{\prime}}, M_{\Theta}, M_{\phi_{I}}$ and $\tan \theta$. When $M_{\phi_{I}}>M_{\Theta}$, the color octet $\Theta$ decays into a pair of gluons, and $\phi_{I}$ decays into $j j \Theta$, so that the final state includes up to six jets, with some of them merged depending on how light the scalars are compared to the coloron.

\footnotetext{
${ }^{2}$ Another gauge extension of QCD without anomalons is $\mathrm{SU}(3)_{1} \times \mathrm{SU}(3)_{2} \times \mathrm{SU}(3)_{3}$ with each generation of SM quarks charged under a different $\mathrm{SU}(3)$ (see also [40] for a possible solution to the strong $\mathcal{C P}$ problem based on this product of gauge groups).

${ }^{3}$ Flavor constraints are stringent [41] in models where the SM quarks transform under different $\mathrm{SU}(3)_{1} \times$ $\mathrm{SU}(3)_{2}$ representations.
} 
When $M_{\phi_{I}}<M_{\Theta}$, the main decays of $\Theta$ are into $q \bar{q} \phi_{I}$ (where $q$ is a SM quark jet), $t \bar{t} \phi_{I}$ or $g g$, while the decays of $\phi_{I}$ have highly suppressed widths. The latter include 3-loop 2body decays (into $W^{+} W^{-}, \gamma Z$ or $Z Z$ ), as well as 1-loop 4-body decays (into $g g q \bar{q}$ or $g g \bar{t} t$ ). The ratio of these widths is estimated in eq. (2.37), where the parameter $\eta_{3}$ introduced in eq. (2.35) accounts for the uncertainty arising from the 3-loop integrals (computing these is beyond the scope of this paper). We expect that $\eta_{3}^{2}$ lies between $O(1)$ and $O\left(10^{-3}\right)$, but even this large uncertainty has a relatively small impact on the possible final states due to the dependence on high powers of the masses (e.g., the ratio of widths is proportional to $\left.M_{\phi_{I}}^{8}\right)$. For $M_{\phi_{I}}$ larger than the mass scale $M_{0}$ [see eq. (4.2)], which is of the order of $1 \mathrm{TeV}$, the main decay is $\phi_{I} \rightarrow q \bar{q} g g$. Although this may be a prompt decay for larger values of $M_{\phi_{I}}$, it may also lead to displaced vertices [see eq. (4.5)]. Final states with top quarks have smaller branching fractions (in some cases only by a factor of $5 / 4$ or $5 / 3$, if there are four or three quark-antiquark pairs) but are still promising. The final states with multiple jets or top quarks are shown in table 1 . For $M_{\phi_{I}} \ll M_{G^{\prime}}$, the $g g q \bar{q}$ system is boosted and forms a jet with 4-prong substructure (labelled by $J_{\phi_{I}}$ ), while the boosted $g g t \bar{t}$ system is even more complicated.

For $M_{\phi_{I}}$ lighter than the mass scale $M_{0}$, the main decay is into a pair of electroweak bosons $\left(W^{+} W^{-}, \gamma Z, Z Z\right)$, and the decay length of $\phi_{I}$ varies between a fraction of millimeter to longer than $1 \mathrm{~cm}$ [see eq. (4.6)]. For $M_{\phi_{I}}<M_{Z}$, the main decay is a 3-loop 3-body decay into a photon and an off-shell $Z$ boson; in that case the decay is likely to be outside the detector, so $\phi_{I}$ would appear as missing transverse energy. The channels with electroweak bosons that have large branching fractions, and which do not rely on fine tuning of masses, are shown in table 2 .

So far there have been dedicated searches for very few of the final states listed in tables 1 and 2. Even though some of these final states can be lumped in certain multi-jet or multi-lepton searches, it is important for the ATLAS, CMS and LHCb collaborations to perform dedicated searches for each of these final states. The sensitivity of a dedicated search far exceeds that of a generic search, especially when the signature involves nested resonances, multi-prong jet substructure, non-isolated leptons or photons, or displaced vertices. Furthermore, depending on the parameter values and the improvements in experimental techniques, any of these signatures may become a discovery mode.

Some of these final states are also encountered in other models. For example, the dominant signals in the ReCoM for a range of masses are $J_{\phi_{I}} j j$ or $J_{\phi_{I}}+4 j$, with a narrow $J_{\phi_{I}}$. These would appear as a $3 j$ or $5 j$ resonance, respectively, which so far have not been searched for. The former is also predicted in models where a vector-like quark is produced at 1-loop in the $s$-channel, from a gluon-quark initial state, and decays via a dimension-6 operator into three SM quarks.

Even dijet resonance searches, if augmented by substructure techniques, may reveal unusual final states, such as $J_{j j \Theta} J_{\Theta}$, in which one jet has a 4-prong substructure and the other jet has a 2-prong substructure and a smaller mass. Other final states are more peculiar, such as those with a large number of electroweak bosons (e.g., $\gamma Z W^{+} W^{-}+4 j$ or $W^{+} W^{-} W^{+} W^{-} t \bar{t} j j$ ), or those involving a displaced $\gamma Z$ resonance. Clearly, there are many new opportunities for new physics searches at the LHC. 


\begin{tabular}{|c|c|c|}
\hline partonic process from $G^{\prime}$ decay & final state & mass relation \\
\hline$\Theta \phi_{I} \rightarrow \Theta(\Theta q \bar{q}) \rightarrow(g g)((g g) q \bar{q})$ & $\begin{array}{c}6 j \\
J_{\Theta} J_{j j \Theta}\end{array}$ & $\begin{array}{l}M_{G^{\prime}}>M_{\phi_{I}}>M_{\Theta} \\
M_{G^{\prime}} \gg M_{\phi_{I}}>M_{\Theta}\end{array}$ \\
\hline$\Theta \phi_{I} \rightarrow\left(\phi_{I} q \bar{q}\right) \phi_{I} \rightarrow((g g q \bar{q}) q \bar{q})(g g q \bar{q})$ & $\begin{array}{c}10 j \\
J_{\phi_{I}}+6 j \\
J_{\phi_{I}} J_{\phi_{I}} j j\end{array}$ & $\begin{array}{l}M_{G^{\prime}}>M_{\Theta}>M_{\phi_{I}} \\
M_{G^{\prime}}-M_{\Theta} \gg M_{\phi_{I}} \\
M_{G^{\prime}}>M_{\Theta} \gg M_{\phi_{I}}\end{array}$ \\
\hline$\Theta \phi_{I} \rightarrow(g g)(g g q \bar{q})$ & $\begin{array}{c}6 j \\
J_{\phi_{I}} j j \\
J_{\Theta} J_{\phi_{I}}\end{array}$ & $\begin{array}{l}M_{G^{\prime}}>M_{\Theta}>M_{\phi_{I}} \\
M_{G^{\prime}}-M_{\Theta} \gg M_{\phi_{I}} \\
M_{G^{\prime}} \gg M_{\Theta}>M_{\phi_{I}}\end{array}$ \\
\hline$\Theta \Theta \rightarrow\left(\phi_{I} q \bar{q}\right)\left(\phi_{I} q \bar{q}\right) \rightarrow((g g q \bar{q}) q \bar{q})((g g q \bar{q}) q \bar{q})$ & $\begin{array}{c}12 j \\
J_{\phi_{I}} J_{\phi_{I}}+4 j\end{array}$ & $\begin{array}{l}M_{\Theta}>M_{\phi_{I}} \\
M_{\Theta} \gg M_{\phi_{I}}\end{array}$ \\
\hline$\Theta \Theta \rightarrow\left(\phi_{I} q \bar{q}\right)(g g) \rightarrow((g g q \bar{q}) q \bar{q})(g g)$ & $\begin{array}{c}8 j \\
J_{\phi_{I}}+4 j\end{array}$ & $\begin{array}{l}M_{\Theta}>M_{\phi_{I}} \\
M_{\Theta} \gg M_{\phi_{I}}\end{array}$ \\
\hline$\Theta \Theta \rightarrow(g g)(g g)$ & $\begin{array}{c}4 j \\
J_{\Theta} J_{\Theta}\end{array}$ & $M_{G^{\prime}} \gg 2 M_{\Theta}$ \\
\hline$\Theta \phi_{I} \rightarrow\left(\phi_{I} q \bar{q}\right) \phi_{I} \rightarrow(g g t \bar{t})(g g q \bar{q}) q \bar{q}$ & $\begin{array}{c}t \bar{t}+8 j \\
J_{g g t \bar{t}} J_{\phi_{I}} j j\end{array}$ & $\begin{array}{l}M_{G^{\prime}}>M_{\Theta}>M_{\phi_{I}} \\
M_{G^{\prime}}>M_{\Theta} \gg M_{\phi_{I}}\end{array}$ \\
\hline & $t \bar{t}+10 j$ & $M_{\Theta}>M_{\phi_{I}}$ \\
\hline$\Theta \Theta \rightarrow\left(\phi_{I} t \bar{t} / q \bar{q}\right)\left(\phi_{I} q \bar{q}\right) \rightarrow(g g q \bar{q} t \bar{t})((g g q \bar{q}) q \bar{q})$ & $\begin{array}{c}J_{g g t \bar{t}} J_{\phi_{I}}+4 j \\
t \bar{t} J_{\phi_{I}} J_{\phi_{I}} j j\end{array}$ & $M_{\Theta} \gg M_{\phi_{I}}$ \\
\hline & $t \bar{t} t \bar{t}+8 j$ & $M_{\Theta}>M_{\phi_{I}}$ \\
\hline$\Theta \Theta \rightarrow\left(\phi_{I} t \bar{t} / q \bar{q}\right)\left(\phi_{I} t \bar{t} / q \bar{q}\right) \rightarrow(g g q \bar{q} t \bar{t})(g g q \bar{q} t \bar{t})$ & $\begin{array}{c}t \bar{t} J_{g g t \bar{t}} J_{\phi_{I}} j j \\
J_{g g t \bar{t}} J_{g g t \bar{t}}+4 j \\
t \bar{t} t \bar{t} J_{\phi_{I}} J_{\phi_{I}} \\
\end{array}$ & $M_{\Theta} \gg M_{\phi_{I}}$ \\
\hline \multirow[b]{2}{*}{$\Theta \Theta \rightarrow\left(\phi_{I} t \bar{t} / q \bar{q}\right)(g g) \rightarrow(g g q \bar{q} t \bar{t})(g g)$} & $t \bar{t}+6 j$ & $M_{\Theta}>M_{\phi_{I}}$ \\
\hline & $\begin{array}{c}J_{g g t \bar{t}}+4 j \\
t \bar{t} J_{\phi_{I}} j j\end{array}$ & $M_{\Theta} \gg M_{\phi_{I}}$ \\
\hline
\end{tabular}

Table 1. Main LHC processes predicted in the ReCoM when $M_{\phi_{I}}>M_{\Theta}$, or when $M_{\phi_{I}}<M_{\Theta}$ with $\phi_{I}$ decaying to $g g q \bar{q}$. Each parenthesis represents a resonance. $J_{\phi_{I}}$ is a jet with 4-prong substructure arising from a boosted $\phi_{I} \rightarrow g g q \bar{q}$, and $J_{g g t \bar{t}}$ is a boosted $\phi_{I} \rightarrow g g t \bar{t}$. Depending on the parameters from eq. (4.5), $J_{\phi_{I}}$ and $J_{g g t \bar{t}}$ may have a displaced vertex for $M_{\phi_{I}} \lesssim 1 \mathrm{TeV}$. $J_{\Theta}$ is a jet with 2-prong substructure due to a boosted $\Theta \rightarrow g g . J_{j j \Theta}$ is a jet with 4-prong substructure due to a boosted $\phi_{I}$. Additional channels are less likely to occur (e.g., $\Theta \Theta \rightarrow J_{j j \phi_{I}} J_{\Theta}$, or $\Theta \phi_{I} \rightarrow 4 t+6 j$ ). 


\begin{tabular}{|c|c|c|}
\hline partonic process from $G^{\prime}$ decay & final state & mass relation \\
\hline$\Theta \phi_{I} \rightarrow\left(\phi_{I} q \bar{q}\right) \phi_{I} \rightarrow((W W) q \bar{q})(W W)$ & $\begin{array}{c}W^{+} W^{-} W^{+} W^{-} j j \\
W^{+} W^{-} J_{W W} j j \\
J_{W W} J_{W W} j j\end{array}$ & $\begin{array}{l}M_{G^{\prime}}>M_{\Theta}>M_{\phi_{I}} \\
M_{G^{\prime}}-M_{\Theta} \gg M_{\phi_{I}} \\
M_{G^{\prime}}>M_{\Theta} \gg M_{\phi_{I}}\end{array}$ \\
\hline$\Theta \phi_{I} \rightarrow\left(\phi_{I} q \bar{q}\right) \phi_{I} \rightarrow(W W)(\gamma Z) q \bar{q}$ & $\begin{array}{c}\gamma Z W^{+} W^{-} j j \\
\gamma Z J_{W W} j j \\
W^{+} W^{-} J_{\gamma Z} j j \\
J_{\gamma Z} J_{W W} j j\end{array}$ & $\begin{array}{c}M_{\Theta}>M_{\phi_{I}} \\
M_{G^{\prime}}-M_{\Theta} \gg M_{\phi_{I}} \\
M_{G^{\prime}}-M_{\Theta} \gg M_{\phi_{I}} \\
M_{\Theta} \gg M_{\phi_{I}}\end{array}$ \\
\hline$\Theta \phi_{I} \rightarrow(g g)(W W)$ & $\begin{array}{c}W^{+} W^{-} j j \\
J_{W W} j j \\
J_{W W} J_{\Theta}\end{array}$ & $\begin{array}{c}M_{\Theta}>M_{\phi_{I}} \\
M_{G^{\prime}}-M_{\Theta} \gg M_{\phi_{I}} \\
M_{G^{\prime}} \gg M_{\Theta}>M_{\phi_{I}}\end{array}$ \\
\hline$\Theta \phi_{I} \rightarrow(g g)(\gamma Z)$ & $\begin{array}{l}\gamma Z j j \\
J_{\gamma Z} j j \\
J_{\gamma Z} J_{\Theta} \\
\end{array}$ & $\begin{array}{c}M_{\Theta}>M_{\phi_{I}} \\
M_{G^{\prime}}-M_{\Theta} \gg M_{\phi_{I}} \\
M_{G^{\prime}} \gg M_{\Theta}>M_{\phi_{I}}\end{array}$ \\
\hline$\Theta \Theta \rightarrow\left(\phi_{I} q \bar{q}\right)\left(\phi_{I} q \bar{q}\right) \rightarrow((W W) q \bar{q})((W W) q \bar{q})$ & $\begin{array}{c}4 W+4 j \\
J_{W W} J_{W W}+4 j\end{array}$ & $\begin{array}{l}M_{\Theta}>M_{\phi_{I}} \\
M_{\Theta} \gg M_{\phi_{I}}\end{array}$ \\
\hline$\Theta \Theta \rightarrow\left(\phi_{I} q \bar{q}\right)\left(\phi_{I} q \bar{q}\right) \rightarrow((\gamma Z) q \bar{q})((W W) q \bar{q})$ & $\begin{array}{c}\gamma Z W^{+} W^{-}+4 j \\
J_{\gamma Z} J_{W W}+4 j\end{array}$ & $\begin{array}{l}M_{\Theta}>M_{\phi_{I}} \\
M_{\Theta} \gg M_{\phi_{I}}\end{array}$ \\
\hline$\Theta \Theta \rightarrow\left(\phi_{I} q \bar{q}\right)(g g) \rightarrow((W W) q \bar{q})(g g)$ & $\begin{array}{c}W^{+} W^{-}+4 j \\
J_{W W}+4 j\end{array}$ & $\begin{array}{l}M_{\Theta}>M_{\phi_{I}} \\
M_{\Theta} \gg M_{\phi_{I}}\end{array}$ \\
\hline$\Theta \Theta \rightarrow\left(\phi_{I} q \bar{q}\right)(g g) \rightarrow((\gamma Z) q \bar{q})(g g)$ & $\begin{array}{l}\gamma Z+4 j \\
J_{\gamma Z}+4 j \\
\end{array}$ & $\begin{array}{l}M_{\Theta}>M_{\phi_{I}} \\
M_{\Theta} \gg M_{\phi_{I}}\end{array}$ \\
\hline$\Theta \Theta \rightarrow\left(\phi_{I} t \bar{t}\right)\left(\phi_{I} q \bar{q}\right) \rightarrow((W W) t \bar{t})((W W) q \bar{q})$ & $\begin{array}{c}4 W+t \bar{t} j j \\
J_{W W} J_{W W} t \bar{t} j j\end{array}$ & $\begin{array}{l}M_{\Theta}>M_{\phi_{I}} \\
M_{\Theta} \gg M_{\phi_{I}}\end{array}$ \\
\hline$\Theta \Theta \rightarrow\left(\phi_{I} t \bar{t}\right)\left(\phi_{I} q \bar{q}\right) \rightarrow(\gamma Z)(W W) t \bar{t} q \bar{q}$ & $\begin{array}{c}\gamma Z W^{+} W^{-} t \bar{t} j j \\
J_{\gamma Z} J_{W W} t \bar{t} j j\end{array}$ & $\begin{array}{l}M_{\Theta}>M_{\phi_{I}} \\
M_{\Theta} \gg M_{\phi_{I}}\end{array}$ \\
\hline
\end{tabular}

Table 2. LHC processes predicted in the ReCoM when $M_{\phi_{I}}<M_{\Theta}$ and $\phi_{I} \rightarrow W W$ or $\gamma Z$. $J_{W W}$ and $J_{\gamma Z}$ represent a $W^{+} W^{-}$or $\gamma Z$ system produced in the decay of a boosted $\phi_{I}$. These originate from a displaced vertex for $M_{\phi_{I}} \lesssim 1 \mathrm{TeV}$, and may appear as $E_{T}$ for $M_{\phi_{I}} \lesssim 100 \mathrm{GeV}$. Additional channels (e.g., those involving two $t \bar{t}$ pairs or $\phi_{I} \rightarrow Z Z$ ) have smaller branching fractions. The mass relations displayed here are necessary but not sufficient (e.g., $\gamma Z J_{W W} j j$ also requires that $M_{\Theta}$ is not much larger than $M_{\phi_{I}}$ ).

\section{Acknowledgments}

We thank Sida Lu, Nhan Tran and Qianfei Xiang for useful discussions and comments. The work of YB is supported by the U.S. Department of Energy under the contract DESC0017647. The work of BD has been supported by Fermi Research Alliance, LLC under Contract No. DE-AC02-07CH11359 with the U.S. Department of Energy, Office of Science, Office of High Energy Physics. 


\section{A Ratios of scalar masses}

We now derive the allowed range for the scalar mass ratio $M_{\phi_{I}} / M_{\Theta}$. As mentioned in section 2 , in the limit $\mu_{\Sigma} \rightarrow 0$, the potential has a global $\mathrm{U}(1)_{\Sigma}$ symmetry, which is broken by the $\Sigma \mathrm{VEV}$. The corresponding Nambu-Goldstone is $\phi_{I}$, so that $M_{\phi_{I}} / M_{\Theta} \rightarrow 0$ for $\mu_{\Sigma} \rightarrow 0$.

The upper limit of $M_{\phi_{I}} / M_{\Theta}$ is more complicated to derive. Let us first use eq. (2.5) to write

$$
\frac{M_{\phi_{I}}}{M_{\Theta}}=\sqrt{3}\left(2+\frac{\sqrt{2} \kappa f_{\Sigma}}{\sqrt{3} \mu_{\Sigma}}\right)^{-1 / 2} .
$$

The necessary and sufficient condition for the potential $V(\Sigma)$ [see eq. (2.1)] to be bounded from below, derived in [11], is

$$
\kappa>\max \{-\lambda,-3 \lambda\}
$$

Imposing this condition, and using the expression of the VEV value $f_{\Sigma}$ in terms of the potential parameters given in eq. (2.3), the scalar mass ratio becomes

$$
\frac{M_{\phi_{I}}}{M_{\Theta}}=\sqrt{3 \lambda+\kappa}\left(2 \lambda+\kappa+\frac{\kappa}{3} \sqrt{4(3 \lambda+\kappa) \frac{m_{\Sigma}^{2}}{\mu_{\Sigma}^{2}}+1}\right)^{-1 / 2} .
$$

The condition (A.2) implies $2 \lambda+\kappa>0$, so that the maximum value of $M_{\phi_{I}} / M_{\Theta}$ occurs at $\kappa<0$ and $m_{\Sigma}^{2}>0$. Furthermore, $m_{\Sigma}^{2} / \mu_{\Sigma}^{2}$ must be maximized to reach the maximum for $M_{\phi_{I}} / M_{\Theta}$. The vacuum that preserves $\mathrm{SU}(3)_{c}$ is the global minimum for $\kappa<0$ (which implies $\lambda>0)$ and $m_{\Sigma}^{2}>0$ provided [11]

$$
(3 \lambda+\kappa) \frac{m_{\Sigma}^{2}}{\mu_{\Sigma}^{2}} \leq \mathcal{G}(\kappa / \lambda),
$$

where the function $\mathcal{G}(x)$ is defined on the interval $-1<x<0$ by

$$
\mathcal{G}(x)=(3+x)\left(\frac{(4+2 x)^{3 / 2}}{\sqrt{1+x}}-2(4+x)\right)^{-1} .
$$

Note that if the inequality (A.4) is not satisfied, then the gauge symmetry breaking pattern in the global minimum is $\mathrm{SU}(3)_{1} \times \mathrm{SU}(3)_{2} \rightarrow \mathrm{SU}(2) \times \mathrm{SU}(2) \times \mathrm{U}(1)$, so color is broken. Nevertheless, an $\mathrm{SU}(3)_{c}$-symmetric local minimum still exists [11] for slightly larger values of $m_{\Sigma}^{2} / \mu_{\Sigma}^{2}$. We will not consider here the possibility that the viable vacuum is not the global minimum.

The constraint (A.4) implies the following upper limit:

$$
\frac{M_{\phi_{I}}}{M_{\Theta}} \leq R_{\max }(\kappa / \lambda)
$$

where the function introduced here is

$$
R_{\max }(x)=\frac{\sqrt{3+x}}{(2+x+(x / 3) \sqrt{4 \mathcal{G}(x)+1})^{1 / 2}} .
$$


For any values of the quartic couplings that satisfy $-1<\kappa / \lambda<0$ we find that the upper limit for the mass ratio is above $\sqrt{3}$. The maximum value $\left(M_{\phi_{I}} / M_{\Theta}\right)_{\max }=3 / \sqrt{2}$ is reached at $\kappa \rightarrow 0$. Hence, the ReCoM predicts

$$
M_{\phi_{I}} \lesssim 2.1 M_{\Theta}
$$

Let us now find the maximum values of $M_{\phi_{I}} / M_{\Theta}$ in other regions of parameter space. For $\kappa \geq 0$ and $m_{\Sigma}^{2}>0$ the only vacuum is $\mathrm{SU}(3)_{c}$ symmetric. From eq. (A.3), the maximum of $M_{\phi_{I}} / M_{\Theta}$ is obtained when $m_{\Sigma}^{2} \rightarrow 0$, so that

$$
\frac{M_{\phi_{I}}}{M_{\Theta}} \leq \sqrt{\frac{3 \lambda+\kappa}{2 \lambda+4 \kappa / 3}} .
$$

The range of $M_{\phi_{I}} / M_{\Theta}$ consistent with a viable global minimum is shown as a function of $\kappa / \lambda$ in figure 15. The solid blue line there is the upper limit for $\kappa<0$, given in (A.6), while the dashed blue line represents the upper limit (A.9) for $\kappa>0$.

For $m_{\Sigma}^{2}<0$, the $\mathrm{SU}(3)_{c}$ symmetric vacuum is the global minimum provided [11]

$$
(3 \lambda+\kappa) \frac{m_{\Sigma}^{2}}{\mu_{\Sigma}^{2}}>-\frac{2}{9} .
$$

If this is not satisfied, the $\mathrm{SU}(3)_{1} \times \mathrm{SU}(3)_{2}$ gauge symmetry is unbroken. If $\kappa<0$, then the maximum is at $m_{\Sigma}^{2} \rightarrow 0$ and given by (A.9). If $\kappa>0$ and $m_{\Sigma}^{2}<0$, then $M_{\phi_{I}} / M_{\Theta}$ reaches its maximum when the above inequality is saturated:

$$
\frac{M_{\phi_{I}}}{M_{\Theta}} \leq \sqrt{\frac{3 \lambda+\kappa}{2 \lambda+10 \kappa / 9}} .
$$

The above upper limit is shown as the solid red lines in figure 15. The conclusion is that even though it is natural to have $M_{\phi_{I}}<M_{\Theta}$, the opposite mass relation, $M_{\phi_{I}}>M_{\Theta}$, also occurs for sizable regions of parameter space.

For the other singlet scalar, $\phi_{R}$, the squared mass given in eq. (2.5) may be rewritten using the expression of the $\Sigma \mathrm{VEV}, f_{\Sigma}$, from (2.3):

$$
M_{\phi_{R}}^{2}=\frac{f_{\Sigma}}{\sqrt{6}} \sqrt{4(3 \lambda+\kappa) m_{\Sigma}^{2}+\mu_{\Sigma}^{2}} .
$$

For $m_{\Sigma}^{2}<0$, the condition (A.10) implies

$$
\frac{1}{3}<\frac{M_{\phi_{R}}}{M_{\phi_{I}}}<\frac{1}{\sqrt{3}}
$$

For $m_{\Sigma}^{2}>0$, the condition (A.2) for the potential to be bounded from below implies a lower limit on $M_{\phi_{R}} / M_{\phi_{I}}$ :

$$
\frac{M_{\phi_{R}}}{M_{\phi_{I}}}>\frac{1}{\sqrt{3}} .
$$

Note that $\phi_{R}$ is much heavier than other scalars when $\lambda \gg|\kappa|$ and $m_{\Sigma}^{2} \gg \mu_{\Sigma}^{2}$. 


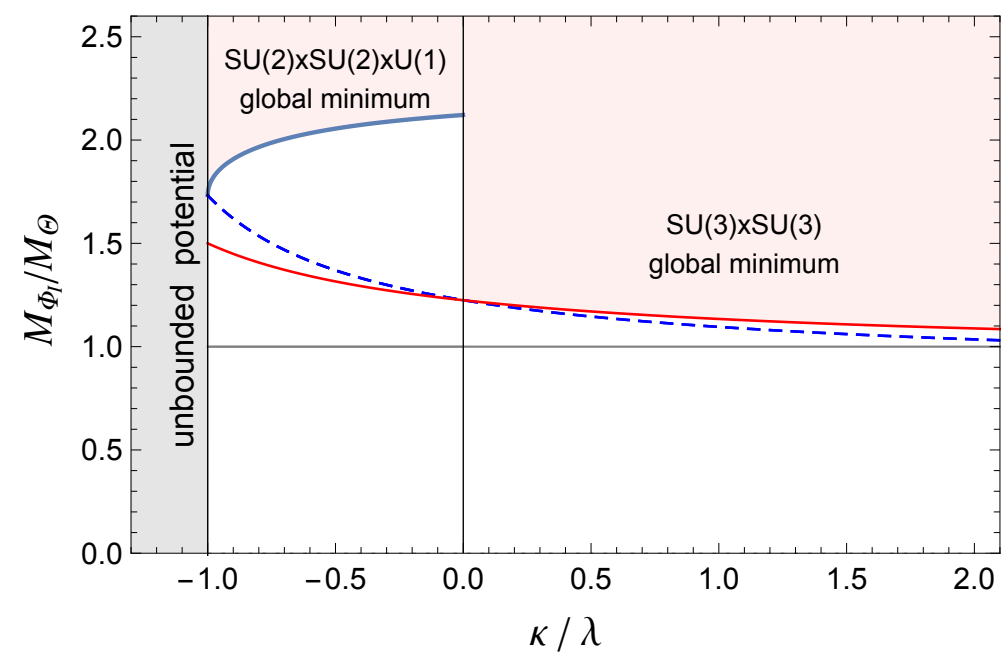

Figure 15. Allowed range (unshaded region) for the scalar mass ratio $M_{\phi_{I}} / M_{\Theta}$ as a function of the quartic coupling ratio $\kappa / \lambda$. In the pink shaded region the global minimum does not preserve color (for $\kappa / \lambda<0$ ) or the extended gauge symmetry is unbroken (for $\kappa / \lambda>0$ ). In the gray shaded region the potential is not bounded from below. The upper limit on $M_{\phi_{I}} / M_{\Theta}$ is given by the solid blue line for $m_{\Sigma}^{2}>0$ and $\kappa<0$; the solid red line for $m_{\Sigma}^{2}>0$ and $\kappa<0$; the dashed blue line for $\kappa m_{\Sigma}^{2}>0$.

\section{B Coloron decays into scalars}

A simple way to compute the partial width of the coloron decay into a pair of color-octet scalars is by fixing the color index of the coloron. Let us compute the width of $G^{\prime 8}$. SU $(3)_{c}$ gauge invariance then ensures that $G^{\prime a}$ has the same width for any $a=1, \ldots, 8$. The interactions of $G^{\prime 8}$ with a pair of color-octet scalars, included in eq. (2.13), is

$$
-\sqrt{3} g_{s} \frac{1-\tan ^{2} \theta}{4 \tan \theta} G_{\mu}^{\prime 8}\left(\Theta^{4} \partial^{\mu} \Theta^{5}-\Theta^{5} \partial^{\mu} \Theta^{4}+\Theta^{6} \partial^{\mu} \Theta^{7}-\Theta^{7} \partial^{\mu} \Theta^{6}\right) .
$$

Here we took into account that the only nonzero values (up to index permutations) of the totally-antisymmetric color tensor $f^{a b c}$ with one index being 8 are $f^{458}=f^{678}=\sqrt{3} / 2$.

The matrix element for $G^{\prime 8} \rightarrow \Theta^{4} \Theta^{5}$ is

$$
\mathcal{M}\left(G^{\prime 8} \rightarrow \Theta^{4} \Theta^{5}\right)=i \sqrt{3} g_{s} \frac{1-\tan ^{2} \theta}{4 \tan \theta}\left(p_{5}^{\mu}-p_{4}^{\mu}\right) \varepsilon_{\mu}(P),
$$

where $\varepsilon_{\mu}$ is the polarization vector of the coloron, and $p_{4}^{\mu}, p_{5}^{\mu}, P^{\mu}$ are the 4-momenta of $\Theta^{4}$, $\Theta^{5}, G^{\prime 8}$, respectively. The matrix element for $G^{\prime 8} \rightarrow \Theta^{6} \Theta^{7}$ has the same form. The two processes do not interfere, so that the squared matrix element for coloron decays into a pair of $\Theta$ scalars, averaged over the coloron polarizations and summed over the two possible final states $\left(\Theta^{4} \Theta^{5}\right.$ and $\left.\Theta^{6} \Theta^{7}\right)$, is given by

$$
\left|\overline{\mathcal{M}}\left(G^{\prime} \rightarrow \Theta \Theta\right)\right|^{2}=g_{s}^{2} \frac{\left(1-\tan ^{2} \theta\right)^{2}}{8 \tan ^{2} \theta}\left(M_{G^{\prime}}^{2}-4 M_{\Theta}^{2}\right) .
$$

This implies that the partial width of the coloron decaying into two $\Theta$ 's is the one given in eq. (2.24). That partial width corrects a factor of 2 missing from the one given in ref. [2]. 
The result in eq. (B.3) can also be checked by using the interaction in eq. (2.13), and summing over all $a, b, c$ indices; when squaring the matrix element, it is necessary to notice that $f^{a b c} \Theta^{b} \partial^{\mu} \Theta^{c}$ can be contracted with both $f^{a b c} \Theta^{b} \partial^{\mu} \Theta^{c}$ and $f^{a c b} \Theta^{c} \partial^{\mu} \Theta^{b}$, which again gives a factor of 2 .

The averaged squared matrix element for the asymmetric decay $G^{\prime} \rightarrow \Theta \phi_{I}$ is slightly more complicated because the scalar masses are different:

$$
\left|\overline{\mathcal{M}}\left(G^{\prime} \rightarrow \Theta \phi_{I}\right)\right|^{2}=g_{s}^{2} \frac{\left(1+\tan ^{2} \theta\right)^{2}}{18 \tan ^{2} \theta M_{G^{\prime}}^{2}}\left[M_{G^{\prime}}^{2}-\left(M_{\Theta}+M_{\phi_{I}}\right)^{2}\right]\left[M_{G^{\prime}}^{2}-\left(M_{\Theta}-M_{\phi_{I}}\right)^{2}\right] .
$$

The corresponding partial width for $G^{\prime} \rightarrow \Theta \phi_{I}$ is given in eq. (2.24), and agrees with the result of ref. [2].

Open Access. This article is distributed under the terms of the Creative Commons Attribution License (CC-BY 4.0), which permits any use, distribution and reproduction in any medium, provided the original author(s) and source are credited.

\section{References}

[1] T.P. Cheng and L.F. Li, Gauge theory of elementary particle physics, Oxford Science Publications, U.K. (1984).

[2] Y. Bai and B.A. Dobrescu, Heavy octets and Tevatron signals with three or four $b$ jets, JHEP 07 (2011) 100 [arXiv: 1012.5814] [INSPIRE].

[3] B.A. Dobrescu and A.D. Peterson, $W^{\prime}$ signatures with odd Higgs particles, JHEP 08 (2014) 078 [arXiv: 1312.1999] [INSPIRE].

[4] C.T. Hill, Topcolor: Top quark condensation in a gauge extension of the standard model, Phys. Lett. B 266 (1991) 419 [INSPIRE].

[5] C.T. Hill and S.J. Parke, Top production: Sensitivity to new physics, Phys. Rev. D 49 (1994) 4454 [hep-ph/9312324] [INSPIRE].

[6] R.S. Chivukula, A.G. Cohen and E.H. Simmons, New strong interactions at the Tevatron?, Phys. Lett. B 380 (1996) 92 [hep-ph/9603311] [INSPIRE].

[7] E.H. Simmons, Coloron phenomenology, Phys. Rev. D 55 (1997) 1678 [hep-ph/9608269] [INSPIRE].

[8] L.J. Hall and A.E. Nelson, Heavy Gluons and Monojets, Phys. Lett. B 153 (1985) 430 [INSPIRE].

[9] P.H. Frampton and S.L. Glashow, Chiral Color: An Alternative to the Standard Model, Phys. Lett. B 190 (1987) 157 [inSPIRE].

[10] J. Bagger, C. Schmidt and S. King, Axigluon Production in Hadronic Collisions, Phys. Rev. D 37 (1988) 1188 [INSPIRE].

[11] Y. Bai and B.A. Dobrescu, Minimal SU(3) $\times \mathrm{SU}(3)$ symmetry breaking patterns, Phys. Rev. D 97 (2018) 055024 [arXiv: 1710.01456] [INSPIRE].

[12] R.S. Chivukula, A. Farzinnia, J. Ren and E.H. Simmons, Constraints on the Scalar Sector of the Renormalizable Coloron Model, Phys. Rev. D 88 (2013) 075020 [Erratum ibid. D 89 (2013) 075020] [arXiv:1307.1064] [INSPIRE]. 
[13] R.S. Chivukula, E.H. Simmons, A. Farzinnia and J. Ren, LHC Constraints on a Higgs boson Partner from an Extended Color Sector, Phys. Rev. D 90 (2014) 015013 [arXiv:1404.6590] [INSPIRE].

[14] E. Nardi, Naturally large Yukawa hierarchies, Phys. Rev. D 84 (2011) 036008 [arXiv:1105.1770] [INSPIRE].

[15] J.R. Espinosa, C.S. Fong and E. Nardi, Yukawa hierarchies from spontaneous breaking of the $\mathrm{SU}(3)_{L} \times \mathrm{SU}(3)_{R}$ flavour symmetry?, JHEP 02 (2013) 137 [arXiv: 1211.6428] [INSPIRE].

[16] E.H. Simmons, Dimension-six Gluon Operators as Probes of New Physics, Phys. Lett. B 226 (1989) 132 [INSPIRE].

[17] L.J. Dixon and Y. Shadmi, Testing gluon selfinteractions in three jet events at hadron colliders, Nucl. Phys. B 423 (1994) 3 [Erratum ibid. B 452 (1995) 724] [hep-ph/9312363] [INSPIRE].

[18] R.S. Chivukula, A. Farzinnia, J. Ren and E.H. Simmons, Hadron Collider Production of Massive Color-Octet Vector Bosons at Next-to-Leading Order, Phys. Rev. D 87 (2013) 094011 [arXiv: 1303.1120] [INSPIRE].

[19] A.D. Martin, W.J. Stirling, R.S. Thorne and G. Watt, Parton distributions for the LHC, Eur. Phys. J. C 63 (2009) 189 [arXiv:0901.0002] [inSPIRE].

[20] CMS collaboration, Searches for dijet resonances in pp collisions at $\sqrt{s}=13 \mathrm{TeV}$ using data collected in 2016., PAS-EXO-16-056 [INSPIRE].

[21] ATLAS collaboration, Search for new phenomena in dijet events using $37 \mathrm{fb}^{-1}$ of $\mathrm{pp}$ collision data collected at $\sqrt{s}=13 \mathrm{TeV}$ with the ATLAS detector, Phys. Rev. D 96 (2017) 052004 [arXiv: 1703.09127] [INSPIRE].

[22] CMS collaboration, Search for $t \bar{t}$ resonances in highly boosted lepton+jets and fully hadronic final states in proton-proton collisions at $\sqrt{s}=13$ TeV, JHEP 07 (2017) 001 [arXiv: 1704.03366] [INSPIRE].

[23] B.A. Dobrescu, K. Kong and R. Mahbubani, Massive color-octet bosons and pairs of resonances at hadron colliders, Phys. Lett. B 670 (2008) 119 [arXiv:0709.2378] [INSPIRE].

[24] R.S. Chivukula, M. Golden and E.H. Simmons, Multi-jet physics at hadron colliders, Nucl. Phys. B 363 (1991) 83 [INSPIRE].

[25] C. Kilic, T. Okui and R. Sundrum, Colored Resonances at the Tevatron: Phenomenology and Discovery Potential in Multijets, JHEP 07 (2008) 038 [arXiv:0802.2568] [INSPIRE].

[26] CMS collaboration, Search for pair-produced dijet resonances in four-jet final states in pp collisions at $\sqrt{s}=7 \mathrm{TeV}$, Phys. Rev. Lett. 110 (2013) 141802 [arXiv:1302.0531] [INSPIRE].

[27] CMS collaboration, Search for pair-produced resonances decaying to jet pairs in proton-proton collisions at $\sqrt{s}=8 \mathrm{TeV}$, Phys. Lett. B 747 (2015) 98 [arXiv:1412.7706] [INSPIRE].

[28] ATLAS collaboration, A search for pair produced resonances in four jets final states in proton-proton collisions at $\sqrt{s}=13 \mathrm{TeV}$ with the ATLAS experiment, ATLAS-CONF-2016-084 [INSPIRE].

[29] ATLAS collaboration, A search for pair-produced resonances in four-jet final states at $\sqrt{s}=13 \mathrm{TeV}$ with the ATLAS detector, ATLAS-CONF-2017-025 [INSPIRE]. 
[30] J.M. Butterworth, A.R. Davison, M. Rubin and G.P. Salam, Jet substructure as a new Higgs search channel at the LHC, Phys. Rev. Lett. 100 (2008) 242001 [arXiv:0802.2470] [INSPIRE].

[31] Y. Bai and J. Shelton, Composite Octet Searches with Jet Substructure, JHEP 07 (2012) 067 [arXiv: 1107.3563] [INSPIRE].

[32] CMS collaboration, Search for Black Holes with Early Run 2 Data, CMS-PAS-EXO-15-007 [INSPIRE].

[33] CMS collaboration, Searches for light- and heavy-flavour three-jet resonances in pp collisions at $\sqrt{s}=8 \mathrm{TeV}$, Phys. Lett. B 730 (2014) 193 [arXiv:1311.1799] [INSPIRE].

[34] ATLAS collaboration, Search for massive supersymmetric particles decaying to many jets using the ATLAS detector in pp collisions at $\sqrt{s}=8$ TeV, Phys. Rev. D 91 (2015) 112016 [Erratum ibid. D 93 (2016) 039901] [arXiv:1502.05686] [INSPIRE].

[35] ATLAS collaboration, Search for massive supersymmetric particles in multi-jet final states produced in pp collisions at $\sqrt{s}=13 \mathrm{TeV}$ using the ATLAS detector at the LHC, ATLAS-CONF-2016-057 [INSPIRE].

[36] CMS collaboration, Inclusive search for new particles decaying to displaced jets at $\sqrt{s}=13 \mathrm{TeV}$, PAS-EXO-16-003 [INSPIRE].

[37] CMS collaboration, Search for long-lived charged particles in proton-proton collisions at $\sqrt{s}=13$ TeV, Phys. Rev. D 94 (2016) 112004 [arXiv:1609.08382] [INSPIRE].

[38] ATLAS collaboration, Search for long-lived, massive particles in events with displaced vertices and missing transverse momentum in $\sqrt{s}=13 \mathrm{TeV}$ pp collisions with the ATLAS detector, Phys. Rev. D 97 (2018) 052012 [arXiv: 1710.04901] [INSPIRE].

[39] ATLAS collaboration, Search for nonpointing and delayed photons in the diphoton and missing transverse momentum final state in $8 \mathrm{TeV}$ pp collisions at the LHC using the ATLAS detector, Phys. Rev. D 90 (2014) 112005 [arXiv: 1409.5542] [INSPIRE].

[40] P. Agrawal and K. Howe, A Flavorful Factoring of the Strong CP Problem, arXiv: 1712.05803 [INSPIRE].

[41] R.S. Chivukula, E.H. Simmons and C.P. Yuan, Axigluons cannot explain the observed top quark forward-backward asymmetry, Phys. Rev. D 82 (2010) 094009 [arXiv:1007.0260] [INSPIRE]. 